\title{
THE TOPOLOGY OF STEIN FILLABLE MANIFOLDS IN HIGH DIMENSIONS I
}

\author{
JONATHAN BOWDEN, DIARMUID CROWLEY, AND ANDRÁS I. STIPSICZ
}

\begin{abstract}
We give a bordism-theoretic characterisation of those closed almost contact $(2 q+1)$-manifolds (with $q \geq 2$ ) which admit a Stein fillable contact structure. Our method is to apply Eliashberg's $h$-principle for Stein manifolds in the setting of Kreck's modified surgery. As an application, we show that any simply connected almost contact 7-manifold with torsion free second homotopy group is Stein fillable. We also discuss the Stein fillability of exotic spheres and examine subcritical Stein fillability.
\end{abstract}

\section{INTRODUCTION}

There have been several recent breakthroughs concerning the existence of contact structures in higher dimensions. In [Bou, GS, [HW, BCS1] contact structures on certain product manifolds, in in [DG2] contact structures on certain $S^{1}$-bundles were constructed and CasalsPancholi-Presas [CPP and Etnyre Et] have shown that every almost contact 5-manifold is contact. The general existence question on higher dimensional manifolds is, however, still open. 1 (In the following we will assume that all almost contact manifolds are closed - for open manifolds the existence question has been settled by using Gromov's $h$-principle.)

Motivated by their 3-dimensional analogues, various notions of fillability and overtwistedness of contact structures on higher dimensional manifolds have been extensively studied, cf. [MNW, MNPS]. The class of contact structures satisfying an appropriate $h$-principle (as overtwisted 3-dimensional contact manifolds do), however, has not yet been identified. 2

In view of this, one is led to consider the existence of contact structures with special properties, the most natural of which is perhaps Stein fillability. Recall that a contact manifold is Stein fillable if it can be realised as the boundary of a Stein domain, which is a compact, complex manifold with boundary admitting a strictly plurisubharmonic function for which the boundary is a regular level set. One of the motivating problems which concerns us here is the following:

Problem 1.1 (Stein Realisation Problem). Determine the almost contact structures which are realised by Stein fillable contact structures.

By Eliashberg's characterisation of Stein manifolds [El1, CE], the existence of Stein fillings in higher dimensions is reduced to a topological question about whether a given manifold admits a nullbordism containing only handles up to the middle dimension and whose tangent bundle admits an almost complex structure. For a given $2 n$-manifold (with $n>2$ ), a direct argument can decide whether it admits a Stein structure, but it is more delicate to see

Date: July 31, 2014.

${ }^{1}$ The recent preprint of Borman, Eliashberg and Murphy [BEM] offers a proof or the statement that any almost contact manifold is contact.

${ }^{2}$ Again, the recent preprint of Borman, Eliashberg and Murphy BEM] contains such a concept. 
whether an odd dimensional manifold can be presented as the boundary of some manifold carrying a Stein structure.

This question can be naturally studied within the framework of (the appropriate) cobordism theory. The main goal of this article is to elucidate the algebro-topological consequences of the above characterisation of Stein fillability by Eliashberg. This approach for constructing contact structures on manifolds was initiated by Geiges [Ge2], and here we pursue the same ideas, using the general setting of Kreck's modified surgery [Kr2].

An almost contact structure $\varphi$ on a closed oriented $(2 q+1)$-manifold $M$ is a reduction of the structure group of the tangent bundle of $M$ to $U(q)$. The almost contact structure $\varphi$ defines a complex structure $\zeta$ on the stable normal bundle of $M$ which we regard as a map $\zeta: M \rightarrow B U$, where $B U$ denotes the classifying space of stable complex vector bundles. The $q^{\text {th }}$ Postnikov factorisation of $\zeta$ consists of a space $B_{\zeta}^{q-1}$ and maps

$$
M \stackrel{\bar{\zeta}}{\longrightarrow} B_{\zeta}^{q-1} \stackrel{\eta_{\zeta}^{q-1}}{\longrightarrow} B U
$$

such that $\eta_{\zeta}^{q-1}$ is a fibration, $\zeta=\eta_{\zeta}^{q-1} \circ \bar{\zeta}$, the map $\bar{\zeta}$ is a $q$-equivalence and $\eta_{\zeta}^{q-1}$ is a $q^{-}$ coequivalence; see Definition 2.11. In addition, there is a canonical bundle isomorphism $\bar{\zeta}^{*}\left(\eta_{\zeta}^{q-1}\right) \cong \nu_{M}$, where $\nu_{M}$ is the stable normal bundle of $M$ (and $\eta_{\zeta}^{q-1}$ is regarded as a stable oriented vector bundle over $\left.B_{\zeta}^{q-1}\right)$. The pair $(M, \bar{\zeta})$ defines a bordism class,

$$
[M, \bar{\zeta}] \in \Omega_{2 q+1}\left(B_{\zeta}^{q-1} ; \eta_{\zeta}^{q-1}\right),
$$

in the bordism theory defined by the complex bundle $\left(B_{\zeta}^{q-1}, \eta_{\zeta}^{q-1}\right)$; see Section 2.1 and Definition 2.9. With these notions in hand we have the following:

Theorem 1.2. A closed almost contact manifold $(M, \varphi)$ of dimension $(2 q+1) \geq 5$ admits a Stein filling if and only if $[M, \bar{\zeta}]=0 \in \Omega_{2 q+1}\left(B_{\zeta}^{q-1} ; \eta_{\zeta}^{q-1}\right)$.

(An expanded version of the result is given in Theorem 3.7). The bordism groups appearing in Theorem 1.2 are isomorphic, via the Pontrjagin-Thom isomorphism, to the stable homotopy groups of the Thom spectrum of $\eta_{\zeta}^{q-1}$. Hence the entire apparatus of stable homotopy theory is available to compute these groups, and if one can show that $\Omega_{2 q+1}\left(B_{\zeta}^{q-1} ; \eta_{\zeta}^{q-1}\right)=0$, a general existence result follows. We will show that this is the case for simply connected 7-manifolds with torsion free second homotopy groups.

Theorem 1.3. Let $M$ be a closed simply connected 7-manifold with $\pi_{2}(M)$ torsion free. Then $M$ admits an almost contact structure, and every almost contact structure on $M$ can be represented by a Stein fillable contact structure.

(Theorem 1.3 can be interpreted as an extension of existence results for contact structures on 1-connected almost contact 5-manifolds and 2-connected 7-manifolds [Ge1, Ge2]).

As expected, the existence of a Stein fillable contact structure on a manifold depends on the smooth structure it carries, and not simply on the underlying homeomorphism type. This fact can be most transparently demonstrated by showing that certain exotic spheres (i.e. smooth manifolds homeomorphic but not diffeomorphic to the sphere of the same dimension) do not carry any Stein fillable contact structures. Using the obstruction class of Theorem 1.2, we prove the following theorem which answers a question raised by Eliashberg, see [El2, 3.8], in roughly three-quarters of all dimensions. 
Theorem 1.4. Let $\Sigma^{2 q+1}$ be a homotopy sphere which admits no framing bounding a paralellizable manifold. If $q \not \equiv 1,3,7 \bmod 8$ or if $q \equiv 1 \bmod 8$ and $q>9$ or if $q=7$ or 15 , then $\Sigma$ admits no Stein fillable contact structure.

(A more precise version of the result is given in Theorem 5.4.)

The obstruction for manifolds to carry Stein fillable contact structures can also be used to establish the following extension of a 3-dimensional result found in Bow to higher dimensions. (The construction is based on non-connected examples of exactly fillable manifolds of [MNW] which are not Stein fillable.)

Theorem 1.5. There exist connected, exactly fillable, contact manifolds that are not Stein fillable in all dimensions greater than three.

Further results (largely concerning $(q-1)$-connected $(2 q+1)$-manifolds) are deferred to a continuation of the present work in BCS2 — in the present paper we emphasize the basic features of the method and restrict ourselves to the applications listed above.

The paper is organized as follows. In Section 2 we give a review of the formulation of Kreck's surgery theory, with the necessary adaptations to the setting of contact and Stein geometry. In particular, we define the obstruction class $[M, \bar{\zeta}]$ of Theorem 1.2 in the appropriate bordism group. In Section 3 we set up notations, recall some basic notions from contact and symplectic topology and interpret the obstruction class defined earlier in the contact context as the obstruction for an almost contact structure to be representable by a Stein fillable contact structure. This result leads to the topological characterization of Stein fillability of Theorem 1.2. As an application, in Section 4 we provide the proof of Theorem 1.3. Section 5 concentrates on highly connected manifolds, and (among other results) we prove Theorem 1.4. In Section 6] we discuss further obstructions for Stein fillability, and prove Theorem [1.5. Finally in Section 7 we formulate a version of the Filling Theorem, Theorem 7.1, which provides an obstruction for subcritical Stein fillability. We also examine the Stein fillability of the product of a contact manifold with a 2-dimensional surface.

Acknowledgements: The authors would like to thank the Max-Planck-Institute in Bonn where parts of this work were carried out and also Anna Abczynski, Fabian Hebestreit and Oscar Randal-Williams for helpful comments. AS was partially supported by OTKA NK81203, by the Lendület program of the Hungarian Academy of Sciences and by ERC LDTBud. The present work is part of the authors' activities within CAST, a Research Network Program of the European Science Foundation.

\section{COMplex MOdified SURGery}

In this section we develop the theory of modified surgery from [Kr2] in the setting where all the stable vector bundles under consideration have complex structures. Before turning to the details, we describe the motivation and the heuristics of the constructions encountered in the section.

The fundamental result underpinning our approach to proving existence results of (Stein fillable) contact structures is the following discovery of Eliashberg [CE, El1] (given formally in Corollary 3.3): A $(2 q+2)$-dimensional compact manifold $W^{2 q+2}$ with boundary $\partial W=M$ and $q \geq 2$ admits the structure of a Stein domain (and hence $M$ admits a Stein fillable contact structure) if and only if $W$ admits (a) an almost complex structure and (b) a handle decomposition involving handles with index at most $q+1$. Therefore in studying the existence 
of Stein fillable contact structures on $M$ we need to understand whether it bounds almost complex manifolds with the required handle decompositions.

Recall that an almost contact structure on $M^{2 q+1}$ is by definition the reduction of the structure group of the tangent bundle to $U(q) \subset S O(2 q+1)$, or equivalently a map $M \rightarrow$ $B U(q)$ covering the classifying map $M \rightarrow B S O(2 q+1)$ of its tangent bundle. Similarly, an almost complex structure on $W^{2 q+2}$ with $\partial W=M$ is simply a map $W \rightarrow B U(q+1)$ covering the classifying map of its tangent bundle. Since computations of bordism groups are best done with stable bundle structures, we will consider stable almost contact (and stable almost complex) structures, hence we need to examine maps from $M$ or $W$ to $B U$. Complex bordism theory (see Example 2.4) tells us that every stably almost contact manifold is the boundary of a stably complex $(2 q+2)$-manifold. (Since Eliashberg's results requires an almost complex structure, rather than a stabilization of it, we need to examine how a stable structure induces an unstable one, and how unique this unstable structure is. This will be explained in Section 2.3.)

The other condition on $W$ for the existence of a Stein structure (regarding a handle decomposition with no handle above the middle dimension) seems less amenable to the techniques of homotopy theory. However, a classic theorem of Wall, (formally stated as Theorem 2.18 in Section 2.4), turns this condition to a problem about homotopy groups. Wall's theorem builds on the $s$-cobordism theorem: Consider $W$ as a handlebody built on $M$, ie. turn the handle decomposition coming from a plurisubharmonic function on $W$ upside down. The lack of high index handles in $W$ implies that this upside down decomposition has no low index handles (ie. handles with indices below the middle dimension). This property then implies that the relative homotopy groups vanish up to the middle dimension. The content of Wall's previously mentioned theorem is the converse of this simple observation: if the relative homotopy groups vanish, a handle decomposition with the required constraint on the indices exists. In turn, the long exact homotopy sequence for the pair $(W, M)$ then implies that the vanishing of the relative groups can be formulated by requiring that the inclusion of $M$ as the boundary induces isomorphism on homotopy groups up to the required dimension. In short, we look for conditions for the existence of $W$ for which the classifying map $\zeta: M \rightarrow B U$ of the stable almost contact structure extends, and for which we have a control on the homotopy groups up to a certain range. The control on the homotopy groups is provided by the following construction of homotopy theory, called Postnikov factorization: for the map $\zeta: M \rightarrow B U$ and an integer $k$ there is a fibration $\eta_{\zeta}^{k}: B_{\zeta}^{k} \rightarrow B U$ and a map $\bar{\zeta}: M \rightarrow B_{\zeta}^{k}$ which factors $\zeta$ into

$$
\zeta=\bar{\zeta} \circ \eta_{\zeta}^{k}
$$

such that $\bar{\zeta}$ induces isomorphisms on the homotopy groups with low index, while $\eta_{\zeta}^{k}$ induces isomorphisms on the homotopy groups with high index. (For the exact statement see Definition 2.11.)

Remark 2.1. The above notion of Postnikov factorization can be found in [Ba, Chapter 5.3], and it is a straighforward generalization of the construction of a Postnikov tower, which is discussed, for example, in [Ha, p. 354]. Indeed, the Postnikov factorizations of the constant map $X \rightarrow\{*\}$ provide the Postnikov tower of $X$.

Considering now the map $\bar{\zeta}: M \rightarrow B_{\zeta}^{k}$ (for appropriately chosen $k$ ), the null-bordism of the pair $(M, \bar{\zeta})$ provides a manifold $W$ which carries a (stable) complex structure, and 
(through the control on its relevant homotopy groups) at the same time admits the required handle decomposition. We point out a further subtlety here: for $f: W \rightarrow B$ providing the null-bordism of $(M, \bar{\zeta})$ we need to require that $f$ also covers the classifying map of the (stable) tangent bundle of $W$. This ensures that the stable almost complex structure lives on the right bundle. Therefore in defining the bordism groups, we are not considering bordism groups of the spaces $B_{\zeta}^{k}$ only, but rather bordism groups with maps to $B U$ and therefore to $B S O$.

The scheme discussed above can be naturally phrased in terms of $(B, \mu)$-manifolds where $\mu: B \rightarrow B S O$ is a stable vector bundle over $B$. We briefly recall this setting in Section 2.1, which allows us to apply Kreck's approach to surgery theory. To be consistent with the existing literature, we will formulate the set-up using stable normal maps, although in our applications we will need almost complex structures on the tangent bundles of even dimensional manifolds. In Section 2.2 we discuss the connection between the stable normal setting and the stable tangential setting and formulate the basic concepts and definitions of "complex modified surgery". In Section 2.3 we handle the transition from stable complex structures to almost complex structures and almost contact structures. After these preliminaries, in Section 2.4 we prove our main surgery lemmas (the Filling Lemma 2.19, and the Stable and Unstable Surgery Lemmas 2.20 and 2.21), which lead to the identification of the obstruction class of Theorem 1.2. (The contact/symplectic interpretation of this class, and hence the proof of Theorem 1.2 will be given in Section 3.) Finally, in Section 2.5 we discuss some explicit constructions and computations in Section 2.5.

2.1. The surgery setting: stable normal bundles. In this subsection we briefly recall the definition of a " $(B, \mu)$-manifold" which is a manifold with extra topological structure on its stable normal bundle. The theory of $(B, \mu)$-manifolds goes back to [La1] and was used systematically in the modified surgery setting of [Kr2]. For a detailed treatment of $(B, \mu)$-manifolds we refer the reader to [Stn, Chapter II] and [Kr2, §2].

The starting point for our discussion of $(B, \mu)$-manifolds is a fibration

$$
\mu: B \rightarrow B S O
$$

where $B S O$ is the classifying space of the stable special orthogonal group $S O$ and $B$ has the homotopy type of a CW complex with a finite number of cells in each dimension. Since $B S O$ classifies oriented stable vector bundles, we regard $(B, \mu)$ as an oriented stable vector bundle over $B$. Given a compact oriented $n$-manifold $X$, let

$$
\nu: X \rightarrow B S O
$$

denote the stable normal Gauss map of $X$. The stable normal Gauss map $\nu$ is defined by the classifying map of the normal bundle of an embedding $X \rightarrow \mathbb{R}^{n+k}$ for $k \gg n$. Letting $k$ tend to infinity, the space of such embeddings is contractible and hence $\nu$ is a well-defined stable vector bundle over $X$.

A $(B, \mu)$-structure on $X$ is a map $\bar{\nu}: X \rightarrow B$ which lifts the map $\nu$ over $\mu$ : that is, there is a commutative diagram:

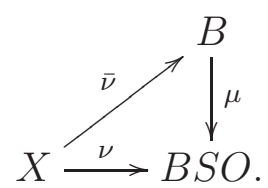

A normal $(B, \mu)$-manifold is a pair $(X, \bar{\nu})$ as above. 
We now give the basic notions needed in the theory of $(B, \mu)$-manifolds.

Equivalence of $(B, \mu)$-structures: Two $(B, \mu)$-structures $\bar{\nu}_{0}$ and $\bar{\nu}_{1}$ on $X$ are equivalent, if there is a $(B, \mu)$-structure $\bar{\nu}$ on $X \times[0,1]$ which restricts to the $(B, \mu)$-structure $\bar{\nu}_{0}$ on $X \times\{0\}$ and to $\bar{\nu}_{1}$ on $X \times\{1\}$.

Pullback of $(B, \mu)$-structures: Given a $(B, \mu)$-structure $\bar{\nu}_{1}: X_{1} \rightarrow B$ and a diffeomorphism $f: X_{0} \cong X_{1}$, there is a canonical pull-back $(B, \mu)$-structure $f^{*}\left(\bar{\nu}_{1}\right)$ on $X_{0}$ that is given by composing all maps with $f$ :

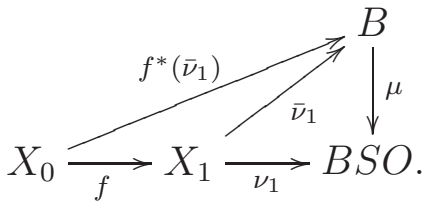

Diffeomorphism of $(B, \mu)$-manifolds: If $\left(X_{0}, \bar{\nu}_{0}\right)$ and $\left(X_{1}, \bar{\nu}_{1}\right)$ are $(B, \mu)$-manifolds, a $(B, \mu)$-diffeomorphism

$$
f:\left(X_{0}, \bar{\nu}_{0}\right) \cong\left(X_{1}, \bar{\nu}_{1}\right)
$$

is a diffeomorphism $f: X_{0} \rightarrow X_{1}$ such that $f^{*}\left(\bar{\nu}_{1}\right)$ and $\bar{\nu}_{0}$ define equivalent $(B, \mu)$-structures on $X_{0}$.

Changing orientations of $(B, \mu)$-manifolds: $\mathrm{A}(B, \mu)$-structure $\bar{\nu}$ on $X$ defines a canonical $(B, \mu)$-structure on $X \times[0,1]$ via pull-back under the projection to $X$, denoted $\pi^{*}(\bar{\nu})$. If $\pi^{*}(\bar{\nu})_{i}:=\left.\pi^{*}(\bar{\nu})\right|_{X \times\{i\}}, i=0,1$, denotes the restriction of $\pi^{*}(\bar{\nu})$ to each end of $X \times[0,1]$, then $\bar{\nu}=\pi^{*}(\bar{\nu})_{1}$ and

$$
-\bar{\nu}:=\left(\pi^{*} \bar{\nu}\right)_{0}=\pi^{*}\left(\left.\bar{\nu}\right|_{X \times\{0\}}\right)
$$

is the $(B, \mu)$-structure defined on the other end of $X \times[0,1]$ via $\pi^{*}(\bar{\nu})$.

Surgery on $(B, \mu)$-manifolds. Let $(X, \bar{\nu})$ be a compact $n$-dimensional $(B, \mu)$-manifold, possibly with boundary, and let $h_{k+1}:=D^{k+1} \times D^{n-k}$ denote an $(n+1)$-dimensional $(k+1)$ handle. For a $(B, \mu) k$-surgery on $(X, \bar{\nu})$ we require the following data:

(1) An embedding $\phi: S^{k} \times D^{n-k} \rightarrow \operatorname{int}(X)$ to the interior of $X$;

(2) A $(B, \mu)$-structure $\bar{\nu}_{W_{\phi}}$ on the trace of surgery on $\phi$,

$$
W_{\phi}:=(X \times I) \cup_{\phi} h_{k+1},
$$

which extends the natural $(B, \mu)$-structure $\bar{\nu} \times I$ on $X \times I \subset X_{\phi}$ induced by $\bar{\nu}$.

The boundary of $W_{\phi}$ is the union $X \cup_{\partial X} X_{\phi}$ where $X_{\phi}$ is the result of surgery on $\phi$. The restriction of the $(B, \mu)$-structure $\bar{\nu}_{W_{\phi}}$ to $X_{\phi}$ is a $(B, \mu)$-structure $\bar{\nu}_{\phi}$ on $X_{\phi}$, giving the $(B, \mu)$-manifold $\left(X_{\phi}, \bar{\nu}_{\phi}\right)$.

Bordism of $(B, \mu)$-manifolds: Suppose that $W$ is an $(n+1)$-manifold with boundary $\partial W=X_{0} \cup_{Y} X_{1}$, that is, $\partial W$ is the union of two compact $n$-manifolds $X_{0}$ and $X_{1}$, both with boundary $Y$. If $\bar{\nu}_{W}: W \rightarrow B$ is a $(B, \mu)$-structure on $W$, then $\bar{\nu}_{W}$ restricts to give $(B, \mu)$ structures $\bar{\nu}_{0}: X_{0} \rightarrow B$ and $\bar{\nu}_{1}: X_{1} \rightarrow B$. In this case $\left(-X_{0},-\bar{\nu}_{0}\right)$ and $\left(X_{1}, \bar{\nu}_{1}\right)$ are called $(B, \mu)$-bordant rel. boundary. If $Y=\phi$ is empty, this gives the defintion of $(B, \mu)$-bordism of closed $(B, \mu)$-manifolds. 
Example $2.2\left((B, \mu)\right.$-diffeomorphism implies $(B, \mu)$-bordism). If $f:\left(X_{0}, \nu_{0}\right) \cong\left(X_{1}, \nu_{1}\right)$ is a $(B, \mu)$-diffeomorphism between closed manifolds then the $s$-cobordism

$$
\left(X_{0} \times[0,1]\right) \cup_{f}\left(X_{1} \times[1,2]\right)
$$

admits the structure of a $(B, \mu)$-bordism between $\left(X_{0}, \bar{\nu}_{0}\right)$ and $\left(X_{1}, \bar{\nu}_{1}\right)$. In particular, closed $(B, \mu)$-diffeomorphic manifolds are $(B, \mu)$-bordant.

Example $2.3((B, \mu)$-bordism and $(B, \mu)$-surgery). By [M, Theorem 1] extended to the case of compact manifolds, every $(B, \mu)$-bordism $\left(W, \bar{\nu}_{W} ; X_{0}, X_{1}\right)$ is $(B, \mu)$-diffeomorphic to the trace of a finite sequence of $(B, \mu)$-surgeries on $\left(X_{0}, \bar{\nu}_{0}\right)$.

The set of $(B, \mu)$-bordism classes of closed $n$-dimensional $(B, \mu)$-manifolds $(N, \bar{\nu})$ form an abelian group

$$
\Omega_{n}(B ; \mu):=\{[N, \bar{\nu}]\}
$$

with addition given by disjoint union and inverse given by $-[M, \bar{\nu}]=[-M,-\bar{\nu}]$.

We give some examples of stable bundles $(B, \mu)$ which we shall use later.

Example 2.4. Consider $(B, \mu)=(B U, F)$ where, if $\pi_{S O}: E S O \rightarrow B S O$ is a model for the universal principal $S O$-bundle, we take $B U$ to be the space $(E S O) / U$, with $U$ acting on $E S O$ via the inclusion $U \hookrightarrow S O$, and $F$ to be the map induced by $\pi_{S O}$. The map $F: B U \rightarrow B O$ is a bundle map with fibre $S O / U$ and corresponds to forgetting almost complex structures on the level of classifying spaces. $(B U, F)$-bordism is then just complex bordism so that $\Omega_{n}(B U ; F)=\Omega_{n}^{U}$. In particular by [Stn, Theorem p.117], we have that $\Omega_{2 q+1}^{U}=0$ and every odd-dimensional stably complex manifold bounds a stably complex manifold.

Example 2.5. $(B, \mu)=\left(B U\langle k+1\rangle, \pi_{k+1}\right)$, where $\pi_{k+1}: B U\langle k+1\rangle \rightarrow B U$ is the $k^{\text {th }}$ connective cover of $B U$ : that is, the universal map such that $\pi_{i}(B U\langle q+1\rangle)=0$ for $i \leq q$ and $\left(\pi_{k+1}\right)_{*}: \pi_{i}(B U\langle k+1\rangle) \cong \pi_{i}(B U)$ for $i \geq k+1$. For example $\left(B U\langle 4\rangle, \pi_{4}\right)=\left(B S U, \pi_{S U}\right)$, where $S U$ denotes the stable special unitary group. In this case $\Omega_{*}\left(B S U ; \pi_{S U}\right)=\Omega_{*}^{S U}$ is special unitary bordism: see [Stn, Chapter X]. By [Stn, p. 248], $\Omega_{9}^{S U} \cong \mathbb{Z}_{2}$ and in Lemma $\underline{5.6}$ we see that the non-zero element in this group is represented by an exotic sphere $\Sigma$ which does not admit a Stein filling.

In general the groups $\Omega_{*}\left(B U\langle k+1\rangle ; \pi_{k+1}\right)$ become harder to compute as $k$ becomes larger: we investigate the groups $\Omega_{2 k+1}\left(B U\langle k\rangle ; \pi_{k}\right)$ and $\Omega_{2 k+1}\left(B U\langle k+1\rangle ; \pi_{k+1}\right)$ further in [BCS2].

Surgery below the middle dimension. For our purposes, the utility of the additional normal structure on $(B, \mu)$-manifolds stems from the following result of Kreck, which we re-state with minor modifications: note that $\left[\frac{n}{2}\right]$ denotes the greatest integer less than or equal to $n / 2$.

Proposition 2.6 ([Kr2, Proposition 4]). Let $\mu: B \rightarrow B S O$ be a fibration and assume that $B$ is connected and has the homotopy type of a $C W$-complex with finite $\left[\frac{n}{2}\right]$-skeleton. Let $\bar{\nu}$ be a normal $(B, \mu)$-structure on an $n$-dimensional compact manifold $X$. Then, if $n \geq 4$, by a finite sequence of $(B, \mu)$-surgeries $(X, \bar{\nu})$ can be replaced by $\left(X^{\prime}, \bar{\nu}^{\prime}\right)$ so that $\bar{\nu}^{\prime}$ is an 
$\left[\frac{n}{2}\right]$-equivalence. In particular, $\left(X^{\prime}, \bar{\nu}^{\prime}\right)$ is again a normal $(B, \mu)$-manifold, i.e. the following diagram commutes:

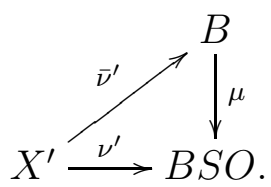

Remark 2.7. We point out that the proof of [Kr2, Proposition 4] contains an error. It uses [Kr2, Lemma 3] which relies on the claim that the higher homotopy groups $\pi_{i}(B)$ are finitely generated over the group ring $\mathbb{Z} \pi$, where $\pi=\pi_{1}(B)$. This is not true in general: see e.g. [Ha, p. 423]. In the proof of [BCS1, Proposition 3.1], which is a modified and specialised version of [Kr2, Proposition 4], we make the same error. However, as we explain below, it is a simple matter to correct the proof using standard methods for surgery below the middle dimension as in [Lü, Lemma 3.55]; see also [Wa4, Theorem 1.2].

Outline of the proof of Proposition 2.6. We work inductively making the maps $\bar{\nu}: X \rightarrow B$ more and more connected. By performing zero surgeries we may assume that $X$ is connected and so $\bar{\nu}$ is an isomorphism on $\pi_{0}$. Since $\pi_{1}(B)$ is finitely generated, further zero-surgeries allow us to make $\bar{\nu}$ surjective on $\pi_{1}$. We then introduce a finite number of relations by doing surgery along a finite collection of embedded curves $(n \geq 4)$ to obtain an isomorphism of fundamental groups: see [Kr2, p. 718]. Hence, we may assume for $1 \leq i<\left[\frac{n}{2}\right]$, that $\bar{\nu}$ is an isomorphism on $\pi_{j}$ for $j \leq i$. From the long exact homotopy sequence,

$$
\cdots \rightarrow \pi_{j}(X) \stackrel{\bar{\nu}_{*}}{\rightarrow} \pi_{j}(B) \rightarrow \pi_{j}(B, X) \rightarrow \pi_{j-1}(X) \rightarrow \ldots,
$$

where we regard $\bar{\nu}$ as an inclusion, it follows that $\pi_{j}(B, X)=0$ for $j \leq i$. The assumption that $B$ has the homotopy type of a $C W$-complex with finite $\left[\frac{n}{2}\right]$-skeleton means that we can apply [Lü, Lemma 3.55] which states that $\pi_{i+1}(B, X)$ is finitely generated over $\mathbb{Z} \pi$ : Here is a short sketch of the proof. Since the pair $(B, X)$ is $i$-connected, the relative Hurewicz Theorem states that $\pi_{i+1}(B, X) \cong H_{i+1}(B, X ; \mathbb{Z} \pi)$. Since $H_{i+1}(B, X ; \mathbb{Z} \pi)$ is the first nontrivial homology group of a chain complex of finitely generated free $\mathbb{Z} \pi$-modules, it is finitely generated.

We choose $\left\{x_{1}, \ldots, x_{k}\right\}$, a set of generators for $\pi_{i+1}(B, X)$. The class $x_{1}$ is represented by a commutative diagram

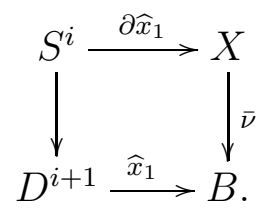

For dimensional reasons we can assume that $\partial \widehat{x}_{1}: S^{i} \rightarrow X$ is an embedding. For any $i$ the stable tangent bundle $\tau_{S^{i}}$ is stably trivial and $\left.\tau_{X}\right|_{\partial \widehat{x}_{1}\left(S^{i}\right)}$ is pulled back from $B$ along a homotopically trivial map, hence $\left.\tau_{X}\right|_{\partial \widehat{x}_{1}\left(S^{i}\right)}=\nu_{\partial \widehat{x}_{1}} \oplus \tau_{S^{i}}$ implies that the normal bundle, $\nu_{\partial \widehat{x}_{1}}$, of $\partial \widehat{x}_{1}\left(S^{i}\right)$ in $X$ is stably trivial. Since the rank of $\nu_{\partial \widehat{x}_{1}}$ is greater than $i$, it follows that $\nu_{\partial \widehat{x}_{1}}$ is trivial and so $\partial \widehat{x}_{1}$ extends to an embedding $\phi_{1}: D^{n-i} \times S^{i} \rightarrow X$. Moreover, the map $\widehat{x}_{1}$ gives the extra data to perform a $(B, \mu)$-surgery on $\phi_{1}$ : see [Lü, Theorem 3.59]. Let $W=(X \times I) \cup h_{i+1}$ be the trace of such a surgery, with normal map $\bar{\nu}_{W}: W \rightarrow B$. The relative group $\pi_{i+1}(W, X)$ is a rank one free $\mathbb{Z} \pi$-module whose generator is mapped to $x_{1}$ 
under the map $\pi_{i+1}(W, X) \rightarrow \pi_{i+1}(B, W)$ induced by the inclusion $i_{X}: X \rightarrow W$. The long exact sequence

$$
\cdots \rightarrow \pi_{i+1}(W, X) \rightarrow \pi_{i+1}(B, X) \rightarrow \pi_{i+1}(B, W) \rightarrow \pi_{i}(X, W) \rightarrow \ldots
$$

shows that $\pi_{i+1}(B, W)$ is generated by $\left\{\left(i_{X}\right)_{*}\left(x_{2}\right), \ldots,\left(i_{X}\right)_{*}\left(x_{k}\right)\right\}$. If $\left(X^{\prime}, \bar{\nu}^{\prime}\right) \subset\left(W, \bar{\nu}_{W}\right)$ denotes the outcome of the $(B, \mu)$-surgery on $\phi_{1}$, then $W \cong\left(X^{\prime} \times I\right) \cup h^{n-i}$ and since $i<\left[\frac{n}{2}\right]$, $n-i>\left[\frac{n}{2}\right]$. From the long exact sequence

$$
\cdots \rightarrow \pi_{j}\left(W, X^{\prime}\right) \rightarrow \pi_{j}\left(B, X^{\prime}\right) \rightarrow \pi_{j}(B, W) \rightarrow \pi_{j-1}\left(X^{\prime}, W\right) \rightarrow \ldots,
$$

we see that $\pi_{j}\left(B, X^{\prime}\right) \cong \pi_{j}(B, W)$ for $j \leq\left[\frac{n}{2}\right]$ and so, by a finite sequence of $(B, \mu)$-surgeries we can achieve that the relative groups $\pi_{j}\left(B, X^{\prime}\right)$ vanish for $j \leq i+1$. Proceeding by induction on $i$, by a finite sequence of $(B, \mu)$-surgeries we can achieve that the relative groups $\pi_{j}\left(B, X^{\prime}\right)$ vanish for $j \leq\left[\frac{n}{2}\right]$; i.e. $\bar{\nu}^{\prime}$ is a $\left[\frac{n}{2}\right]$-equivalence.

2.2. Stable complex structures. An example of $(B, \mu)$-manifolds of particular interest in this paper (recall Example 2.4) is given by

$$
(B, \mu)=(B U, F)
$$

where $F: B U \rightarrow B O$ is the canonical forgetful map between classifying spaces. A ( $B U, F)$ manifold is nothing but a stably complex manifold. Notice that an almost complex structure $J$ on a $2 q$-manifold $X$ (that is, a reduction of the structure group of the tangent bundle of $X$ from $S O(2 q)$ to $U(q)$ ) naturally induces a stable complex structure on $\tau_{X}$, the stable tangent bundle of $X$. As there is a canonical bundle isomorphism,

$$
\tau_{X} \oplus \nu_{X} \cong \varepsilon
$$

where $\nu_{X}$ is the stable normal bundle of $X$ and $\varepsilon$ denotes the trivial stable bundle, a stable complex structure on $\tau_{X}$ induces a stable complex structure on $\nu_{X}$ : choose the unique stable complex structure on $\nu_{X}$ so that the sum with the given stable complex structure on $\tau_{X}$ is the trivial stable complex structure on $\varepsilon$. We shall denote the stable normal complex structure associated to $(X, J)$ by $S J$ or sometimes $\zeta_{X}$.

As in the even-dimensional case, an almost contact structure $\varphi$ on a closed $(2 q+1)$-manifold $M$ (that is, the reduction of the structure group from $S O(2 q+1)$ to $U(q)$ ) induces a stable complex structure $S \varphi=\zeta_{M}$ on the stable normal bundle of $M$. (We will also call the stabilized structures complex rather than contact in the odd-dimensional case.) Since stable tangential complex and stable normal complex structures determine each other, we will focus on the normal picture (although in the applications we will need results for the tangential structures).

Building on the discussion of $(B, \mu)$-manifolds from Section 2.1, we now establish the basic notions in stable complex surgery which we shall use throughout this paper. Let

$$
\eta: B \rightarrow B U
$$

be a fibration, where, as before, $B$ has the homotopy type of a CW complex with a finite number of cells in each dimension. We regard $(B, \eta)$ as a stable complex vector bundle over $B$ with underlying oriented bundle $F \circ \eta: B \rightarrow B S O$. We shall be interested in the situation 
described by the following commutative diagram:

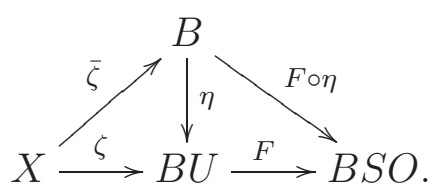

Here $X$ is an oriented manifold with stable normal bundle $\nu=F \circ \zeta,(X, \zeta)$ is a compatibly oriented stably complex manifold and $(X, \bar{\zeta})$ is a $(B, F \circ \eta)$-manifold. Since $F$ is fixed, we shall usually call $(X, \bar{\zeta})$ a $(B, \eta)$-manifold for short: this simply means that $(X, \bar{\zeta})$ is a $(B, F \circ \eta)$-manifold.

Definition 2.8 ( $\zeta$-compatible $(B, \eta)$-manifold). In the situation of the commutative diagram (11) above, we say that $\bar{\zeta}: X \rightarrow B$ is a $\zeta$-compatible $(B, \eta)$-manifold; i.e., $(X, \bar{\zeta})$ is a $(B, F \circ \eta)$ manifold with underlying stably complex manifold $(X, \zeta)$.

It follows from the definitions that $(-X,-\bar{\zeta})$ is a $(-\zeta)$-compatible $(B, \eta)$-manifold and that a $(B, F \circ \eta)$-diffeomorphism $f:\left(X_{0}, \bar{\zeta}_{0}\right) \cong\left(X_{1}, \bar{\zeta}_{1}\right)$ is also a stably complex diffeomorphism $f:\left(X_{0}, \eta \circ \zeta_{0}\right) \cong\left(X_{1}, \eta \circ \zeta_{1}\right) .(B, \eta)$-bordism groups are defined as follows.

Definition 2.9 (( $B, \eta)$-bordism). We define

$$
\Omega_{n}(B ; \eta):=\Omega_{n}(B ; F \circ \eta)
$$

to be the bordism group of $(B, F \circ \eta)$-bordism classes of closed $n$-dimensional $(B, F \circ \eta)$ manifolds as defined in Section 2.1.

The following definition (as explained at the beginning of this section) is of fundamental importance in our present discussion.

Definition 2.10 (Normal $k$-smoothing). A normal $k$-smoothing in $(B, \eta)$ is a normal $(B, \eta)$ manifold $(X, \bar{\zeta})$, where $\bar{\zeta}: X \rightarrow B$ is a $(k+1)$-equivalence; i.e. $\bar{\zeta}$ induces an isomorphism on homotopy groups $\pi_{i}$ for $i \leq k$ and a surjection on $\pi_{k+1}$.

For the purposes of understanding Stein fillings, we shall be interested in the case where $\bar{\zeta}$ is a $k$-smoothing for certain $k$. One may ask whether for some stably complex manifold $(X, \zeta)$ there are any $\zeta$-compatible normal $k$-smoothings $\bar{\zeta}: X \rightarrow B$ at all. In fact this is always the case because the map $\zeta: X \rightarrow B U$ can be factorised (up to homotopy) as a composition

$$
X \stackrel{\bar{\zeta}}{\longrightarrow} B_{\zeta}^{k} \stackrel{\eta_{\zeta}^{k}}{\longrightarrow} B U
$$

where $\bar{\zeta}$ is a $(k+1)$-equivalence and $\eta_{\zeta}^{k}$ is a fibration. The space $B_{\zeta}^{k}$ and the maps $\bar{\zeta}$ and $\eta_{\zeta}^{k}$ make up the $k^{\text {th }}$ Postnikov factorisation of $\zeta$. The existence of the $k^{\text {th }}$ Postnikov factoriation is proven in [Ba, Theorem 5.3.1] and [Ha, Proposition 4.13], its defining properties are identified in Definition 2.11 below and we discuss some examples in Section 2.5. In general, for any $k \geq 0$, a map $f: X \rightarrow Y$ between $C W$-complexes, has a $k^{t h}$ Postnikov factorisation $f \simeq \eta_{f}^{k} \circ \bar{f}$ by maps $\bar{f}: X \rightarrow Y_{f}^{k}$ and $\eta^{k}: Y_{f}^{k} \rightarrow Y$. Such factorisations are built by first converting $f$ into a fibration and then working inductively so that there are fibrations $Y_{f}^{k} \rightarrow Y_{f}^{k-1}$ with fibre $K\left(\pi_{k}(F), k\right)$ where $F$ is the homotopy fibre of $f: X \rightarrow Y$. 
Definition 2.11 (Complex normal $k$-type). Let $(X, \zeta)$ be a stably complex manifold. The complex normal $k$-type of $(X, \zeta)$, denoted $\left(B_{\zeta}^{k}, \eta_{\zeta}^{k}\right)$, is defined to be the fibre homotopy type of the fibration $\eta_{\zeta}^{k}$ in the following diagram:

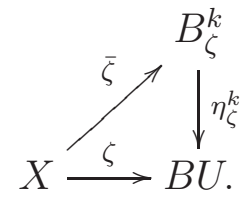

The fibration $\eta_{\zeta}^{k}$ is uniquely defined up to fibre homotopy type by the following properties:

(1) the map $\bar{\zeta}$ is a $(k+1)$-equivalence,

(2) the map $\eta_{\zeta}^{k}$ is a $(k+1)$-coequivalence, i.e. $\left(\eta_{\zeta}^{k}\right)_{*}: \pi_{j}\left(B_{\zeta}^{k}\right) \rightarrow \pi_{j}(B U)$ is injective when $j=k+1$ and an isomorphism if $j>k+1$.

We conclude this subsection by considering the role of the choice of the normal $(q-1)$ smoothing $\bar{\zeta}: X \rightarrow B$ on the bordism class $[X, \bar{\zeta}] \in \Omega_{2 q+1}(B: \eta)$. Our method is to adapt the key point of the proof of [Kr1, Proposition 7.4] to the complex setting. Given a stable complex vector bundle $\eta: B \rightarrow B U$, let $\operatorname{Aut}(B, \eta)$ be the group of fibre homotopy classes of fibre self-homotopy equivalences of $\eta$. That is, $\operatorname{Aut}(B, \eta)$ consists of fibre homotopy equivalence classes of maps $\alpha: B \simeq B$ which make the following diagram commute:

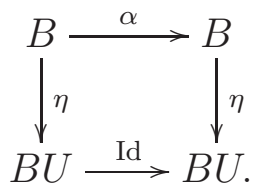

The group $\operatorname{Aut}(B, \eta)$ acts on the set of $(B, \eta)$-diffeomorphism classes of complex normal $k$ smoothings in $(B, \eta)$ by mapping a complex normal $k$-smoothing $\bar{\zeta}: X \rightarrow B$ to the complex normal $k$-smoothing $\alpha \circ \bar{\zeta}: X \rightarrow B$.

Lemma 2.12 (cf. [Kr1, Proposition 7.4]). Suppose that $\left(X_{0}, \zeta_{0}\right)$ and $\left(X_{1}, \zeta_{1}\right)$ are stably complex manifolds and that for $i=0,1, \bar{\zeta}_{i}: X_{i} \rightarrow B_{\zeta_{0}}^{k}$ is a $\zeta_{i}$-compatible normal $k$-smoothing in $\left(B_{\zeta_{0}}^{k}, \eta_{\zeta_{0}}^{k}\right)$, the complex normal $k$-type of $\left(X_{0}, \zeta_{0}\right)$. If $f:\left(X_{0}, \zeta_{0}\right) \cong\left(X_{1}, \zeta_{1}\right)$ is a stably complex diffeomorphism, then there is a fibre homotopy self-equivalence $\alpha \in \operatorname{Aut}\left(B_{\zeta_{0}}^{k}, \eta_{\zeta_{0}}^{k}\right)$ such that $f$ is a $\left(B_{\zeta_{0}}^{k}, \eta_{\zeta_{0}}^{k}\right)$-diffeomorphism from $\left(X_{0}, \alpha \circ \bar{\zeta}_{0}\right)$ to $\left(X_{1}, \bar{\zeta}_{1}\right)$.

Proof. The maps

$$
\bar{\zeta}_{0}, f^{*}\left(\bar{\zeta}_{1}\right): X_{0} \rightarrow B_{\zeta_{0}}^{k}
$$

determine two complex normal $k$-smoothings on $X_{0}$. Now the universal properties of Postnikov stages of maps [Ba, Corollary 5.3.8] ensure that there is a fibre homotopy equivalence $\alpha:\left(B_{\zeta_{0}}^{k}, \eta_{\zeta_{0}}^{k}\right) \simeq\left(B_{\zeta_{0}}^{k}, \eta_{\zeta_{0}}^{k}\right)$ such that $\alpha \circ \bar{\zeta}_{0}$ and $f^{*}\left(\bar{\zeta}_{1}\right)$ are equivalent $\left(B_{\zeta_{0}}^{k}, \eta_{\zeta_{0}}^{k}\right)$-structures on $X_{0}$. By definition, this means that $f$ is a $\left(B_{\zeta_{0}}^{k}, \eta_{\zeta_{0}}^{k}\right)$-diffeomorphism from $\left(X_{0}, \alpha \circ \bar{\zeta}_{0}\right)$ to $\left(X_{1}, \bar{\zeta}_{1}\right)$.

The following corollary is an important consequence of Lemma 2.12 which arises from the fact that the induced action of $\operatorname{Aut}\left(B_{\zeta}^{k}, \eta_{\zeta}^{k}\right)$ on $\Omega_{n}\left(B_{\zeta}^{k} ; \eta_{\zeta}^{k}\right)$ is by group automorphisms.

Corollary 2.13. Let $\left(B_{\zeta}^{k}, \eta_{\zeta}^{k}\right)$ be the normal $k$-type of $(X, \zeta)$. If $\bar{\zeta}: X \rightarrow B_{\zeta}^{k}$ is a closed $\zeta$ compatible normal $k$-smoothing such that $[X, \bar{\zeta}]=0 \in \Omega_{n}\left(B_{\zeta}^{k} ; \eta_{\zeta}^{k}\right)$, then for all $\zeta$-compatible normal $k$-smoothings $\hat{\zeta}: X \rightarrow B_{\zeta}^{k}$ we have that $[X, \hat{\zeta}]=0 \in \Omega_{n}\left(B_{\zeta}^{k} ; \eta_{\zeta}^{k}\right)$. 
Proof. Applying Lemma 2.12 to $(X, \bar{\zeta})$ and $(X, \hat{\zeta})$ we deduce that there is a fibre homotopy equivalence $\alpha:\left(B_{\zeta}^{k}, \eta_{\zeta}^{k}\right) \simeq\left(B_{\zeta}^{k}, \eta_{\zeta}^{k}\right)$ such that $(X, \hat{\zeta})$ is $\left(B_{\zeta}^{k}, \eta_{\zeta}^{k}\right)$-diffeomorphic to $(X, \alpha \circ \bar{\zeta})$. Hence $[X, \hat{\zeta}]=\alpha_{*}([X, \bar{\zeta}])=0$.

2.3. Stable and unstable surgery. Propagating contact structures over Weinstein handles requires information about almost contact structures of $M$. On the other hand, computations in $(B, \eta)$-bordism require the use of the stable normal bundle of $M$. In this subsection we prove two lemmas which allow us to move between these two settings.

Recall that $h_{k+1}:=D^{k+1} \times D^{n-k}$ is an $(n+1)$-dimensional $(k+1)$-handle. Let $(M, \varphi)$ be a closed $(2 q+1)$-dimensional almost contact manifold and set $n:=2 q+1$. For an almost complex $k$-surgery on $(M, \varphi)$ we require the following data:

(1) An embedding $\phi: S^{k} \times D^{n-k} \rightarrow M$;

(2) An almost complex structure $J$ on

$$
W_{\phi}:=(M \times I) \cup_{\phi} h_{k+1},
$$

extending the natural almost complex structure $\varphi \times I$ on $M \times I \subset W_{\phi}$ induced by $\varphi$. The result of this surgery is the other boundary component of $W_{\phi}$, denoted $M_{\phi}$. It is an almost contact manifold with almost contact structure $\varphi_{\phi}:=\left.J\right|_{M_{\phi}}$.

Definition 2.14 (Almost complex surgery). In the situation above, we shall say that the almost contact manifold $\left(M_{\phi}, \varphi_{\phi}\right)$ is obtained from $(M, \varphi)$ by a $k$-dimensional almost complex surgery.

When we work with stably complex manifolds, we have the analogous situation, where almost contact structures and almost complex structures on the tangent bundle are replaced first by stable complex structures on the tangent bundle and then by stable complex structures on the normal bundle. Thus to perform stable complex $k$-surgery on a stably complex $n$-manifold $(M, \zeta)$, we require an embedding $\phi: S^{k} \times D^{n-k} \rightarrow M$ along with an extension of the stable complex structure $\zeta$ to a stable complex structure on the trace of the surgery $W_{\phi}=(M \times I) \cup_{\phi} h_{k+1}$. In this case $M_{\phi}$ inherits the stable complex structure $\zeta_{\phi}:=\left.\zeta\right|_{M_{\phi}}$, and we shall say that $\left(M_{\phi}, \zeta_{\phi}\right)$ is obtained from $(M, \zeta)$ via stable complex surgery.

Notation 2.15. Given an almost contact manifold $(M, \varphi)$, let $(M, \zeta)$ denote the stably complex manifold defined by $\varphi$.

Lemma 2.16. Let $(M, \varphi)$ be a $(2 q+1)$-dimensional almost contact manifold and suppose $\left(W, \zeta_{W} ; M, M_{\phi}\right)$ is the trace of a stable complex $k$-surgery on $(M, \zeta)$ with $k \leq 2 q$. Then there is an almost complex structure $J$ on $W$ with $S J=\zeta_{W}$ and which restricts to $\varphi \times I$ on $M \times I$.

(Notice that the above lemma is stated for $k \leq 2 q$ - in our applications, however, we will use the statement only in the range $k \leq q+1$.)

Proof. First we notice that the stable normal complex structure on $W$ can be converted to a stable tangential complex structure on $W$ which stabilies $\varphi \times I$ when restricted to $M \times I$. Our problem is to reduce the structure group of the tangent bundle of $W$ to $U(q+1)$, and we must do this relative to the chosen reduction corresponding to $\varphi \times I$ on $M \times I$. We encounter 
the unstable lifting problem which maps to the stable lifting problem:

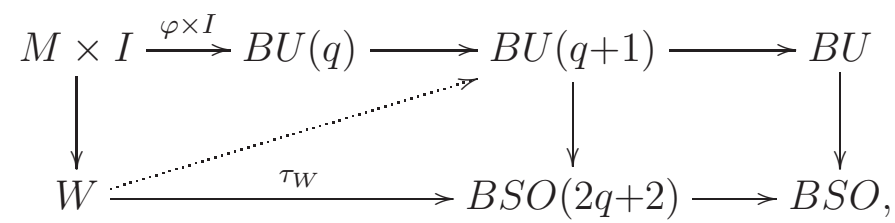

where $\tau_{W}$ classifies the tangent bundle of $W$. The lifting obstructions for these problems lie in the groups

$$
H^{k+1}\left(W, M ; \pi_{k}(S O(2 q+2) / U(q+1))\right) \quad \text { and } \quad H^{k+1}\left(W, M ; \pi_{k}(S O / U)\right),
$$

and the unstable lifting obstruction maps to the stable lifting obstruction under the coefficient homomorphism $S_{*}: \pi_{k}(S O(2 q+2) / U(q+1)) \rightarrow \pi_{k}(S O / U)$. The map $S_{*}$ is an isomorphism for $k \leq 2 q$ by [Gr, p. 432]. Hence the vanishing of the stable obstruction ensures the vanishing of the unstable obstruction. It follows therefore that there is an almost complex structure $J$ on $W$ compatible with $(M \times I, \varphi \times I)$.

Note that in the setting of Lemma 2.16 there may be several homotopy classes of almost contact structures $\varphi^{\prime}$ on $M_{\phi}$ such that $S \varphi^{\prime}$ is homotopic to $\zeta_{\phi}$. The almost complex structure $J$ will induce one such structure $\left.J\right|_{M_{\phi}}$ on $M_{\phi}$. To obtain an almost complex bordism $\left(W, J ;(M, \varphi),\left(M_{\phi}, \varphi^{\prime}\right)\right)$ for a specific almost contact structure $\varphi^{\prime}$ we may need to find an alternative bordism.

Lemma 2.17. Let $(M, \zeta)$ be a closed connected stably complex $(2 q+1)$-manifold.

(1) There is an almost contact structure $\varphi$ such that $\zeta=S \varphi$.

(2) Suppose that two almost contact structures $\varphi$ and $\varphi^{\prime}$ are such that $S \varphi=S \varphi^{\prime}=\zeta$. Then there is an almost contact structure $\varphi_{d}$ on the sphere $S^{2 q+1}$ such that:

(a) $(M, \varphi) \#\left(S^{2 q+1}, \varphi_{d}\right)$ and $\left(M, \varphi^{\prime}\right)$ are equivalent as almost contact manifolds,

(b) $S \varphi_{d}=S \varphi_{0}$ where $\varphi_{0}$ is the standard almost contact structure on $S^{2 q+1}$,

(c) $\left(S^{2 q+1}, \varphi_{d}\right)$ bounds an almost complex $(2 q+2)$-manifold with a handle decomposition of handles of indices $\leq q+1$.

Proof. Following Gray [Gr, p. 432], we write

$$
F_{2 q+1}=S O(2 q+1) / U(q) \text { and } F_{2 q+2}=S O(2 q+2) / U(q+1) .
$$

Gray [Gr, p. 432] shows that there is a homeomorphism $F_{2 q+1} \cong F_{2 q+2}$ and a fibre bundle

$$
F_{2 q+1} \rightarrow F_{2 q+3} \rightarrow S^{2 q+2}
$$

As Sato [Sa, Proposition 1] observes, the homeomorphism $F_{2 q+1} \cong F_{2 q+2}$ entails that there is a bijection between the set of homotopy classes of almost contact structures on $M$ and the set of homotopy classes of almost complex structures on $M \times I$. Since there is also a bijection between the sets of homotopy classes of stable complex structures on $M$ and on $M \times I$, it suffices to prove that every stable complex structure on $M \times I$ is the stabilisation of an almost complex structure on $M \times I$. But this last point is true for dimensional reasons, as we now explain.

Let $V$ be any oriented vector bundle of real rank $2 j$ over a space $X$ which is homotopy equivalent to a closed connected oriented $(2 j-1)$-manifold. Let $F(V) \rightarrow X$ and $F^{S}(V) \rightarrow X$ be, respectively the bundle of oriented, orthogonal frames and its stable analogue: $F(V)$ is 
a principal $S O(2 j)$-bundle over $X$ and $F^{S}(V)$ is a principal $S O$ bundle over $X$. In addition, we define $\mathcal{J}(V):=F(V) / U(j)$ and $\mathcal{J}^{S}(V):=F^{S}(V) / U$ and the stabilisation map

$$
S: \mathcal{J}(V) \rightarrow \mathcal{J}^{S}(V) \text {. }
$$

A complex structure on $V$ corresponds to a section of the $S O(2 j) / U(j)$-bundle $\mathcal{J}(V) \rightarrow X$, a homotopy of complex structures corresponds to a homotopy of sections and a similar statement holds for stable complex structures on $V$ and sections of the $S O / U$-bundle $\mathcal{J}^{S}(V) \rightarrow X$. To compare stable and unstable complex structures on $V$ we consider the following diagram:

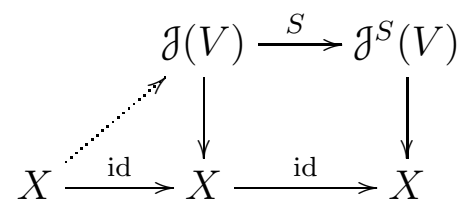

Note that the homeomorphism $F_{2 j-1} \cong F_{2 j}$ and the fibre bundle (2) show that the stabilisation map $S: F_{2 j} \rightarrow S O / U$ is a $(2 j-1)$-equivalence. Let $X^{(2 j-2)} \subset X$ be a $(2 j-2)$-skeleton. Elementary obstruction theory applied to the lifing problem above shows the following:

(1) Every section $\sigma^{S}$ of $\mathcal{J}^{S}(V) \rightarrow X$ is homotopic to some stabilisation $S \circ \sigma$ where $\sigma$ is a section of $\mathcal{J}(V) \rightarrow X$.

(2) If $\sigma$ and $\sigma^{\prime}$ are sections of $\mathcal{J}(V) \rightarrow X$ such that $S \circ \sigma$ and $S \circ \sigma^{\prime}$ are homotopic, then there is a homotopy $H$ between the restrictions $\left.\sigma\right|_{X^{(2 j-2)}}$ and $\left.\sigma^{\prime}\right|_{X^{(2 j-2)}}$. Moreover, the homotopy $H$ defines an obstruction class,

$$
\mathfrak{o}_{H} \in H^{2 j}\left(X ; A_{2 j-1}\right),
$$

where $A_{2 j-1}:=\operatorname{ker}\left(\pi_{2 j-1}(S O(2 j) / U(j)) \rightarrow \pi_{2 j-1}(S O / U)\right)$, and if $\mathfrak{o}_{H}=0$, then $\sigma$ and $\sigma^{\prime}$ are homotopic.

This proves (1) and parts (a) and (b) of (2).

We now turn to the proof of (2) (c). The homotopy classes of almost contact structures on $S^{2 q+1}$ which are stably equivalent to the standard almost contact structure $\varphi_{0}$ are parametrised by the group $A_{2 q+1}$ define above. As noted in [Ge2, p. 1201], results of Sato [Sa], building on work of Morita, show that for $q$ even one can realise all these homotopy classes $\varphi$ via contact structures on various standard Brieskorn spheres and that all of these $\varphi$ are Stein fillable. The Stein fillings then provide the required almost complex $(2 q+2)$-manifolds.

A similar observation holds when $q$ is odd, by utilising the calculations of [DG1]. One simply takes the product of even dimensional spheres $W=S^{q+1} \times S^{q+1}$. The stable tangent bundle of $W$ is trivial, so we choose a stable trivialisation. After removing a ball we obtain a stably complex filling of $S^{2 q+1}$ which is built of two $(q+1)$-handles and a zero-handle. Thus the stable almost complex structure determines a unique complex structure $J^{\bullet}$ on $W^{\bullet}=W \backslash \operatorname{int}\left(D^{2 q+2}\right)$. Using the notation of [DG1] we have $\mathfrak{o}\left(W^{\bullet}, J^{\bullet}\right)=2$ and the formulae of [DG1, p. 3831] allow one to realise all possible unstable almost contact structures in any equivalence class of stable almost contact structures via connect sums with $\partial W^{\bullet}$.

2.4. The surgery lemmas. In this subsection $\eta: B \rightarrow B U$ is again a stable complex vector bundle. The two lemmas below are consequences of the following classical theorem of Wall:

Theorem 2.18 ([Wa3], Theorem 3). Suppose that $W$ is a (connected) $n$-dimensional cobordism from $X_{-}$to $X_{+}$and assume that $\left(W, X_{-}\right)$is $r$-connected, that is, the relative homotopy groups $\pi_{i}\left(W, X_{-}\right)$vanish for $i \leq r$. Suppose furthermore that $r \leq n-4$. Then, the cobordism 
is geometrically $r$-connected, that is, $W$ admits a (relative) handle decomposition built on $X_{-}$ which involves no handle with index $\leq r$.

In particular, Wall's result converts a potentially subtle problem about Morse functions with certain properties to a question that is purely homotopy theoretic in nature.

Lemma 2.19 (Filling lemma). Let $(M, \varphi)$ be an almost contact $(2 q+1)$-manifold with induced stable complex structure $\zeta$ and complex normal $(q-1)$-type $\left(B_{\zeta}^{q-1}, \eta_{\zeta}^{q-1}\right)$. If $q \geq 2$, then the following are equivalent:

(1) $(M, \varphi)$ is the boundary of a compact almost complex $(2 q+2)$-manifold $(W, J)$ with handles only of index $q+1$ and smaller.

(2) For any $\zeta$-compatible normal $(q-1)$-smoothing $\bar{\zeta}: M \rightarrow B_{\zeta}^{q-1}$, we have

$$
[M, \bar{\zeta}]=0 \in \Omega_{2 q+1}\left(B_{\zeta}^{q-1} ; \eta_{\zeta}^{q-1}\right)
$$

(3) For some stable complex bundle $(B, \eta)$ and some $\zeta$-compatible normal (q-1)-smoothing $\bar{\zeta}: M \rightarrow B$, we have

$$
[M, \bar{\zeta}]=0 \in \Omega_{2 q+1}(B ; \eta) .
$$

Proof. $(1) \Rightarrow(2)$ : Suppose that $(W, J)$ is as in the statement of the lemma. The almost complex structure $J$ defines a stable complex structure $\zeta_{W}: W \rightarrow B U$. Let $\left(B_{\zeta_{W}}^{q-1}, \eta_{\zeta_{W}}^{q-1}\right)$ be the complex normal $(q-1)$-type of $\left(W, \zeta_{W}\right)$ and let $\bar{\zeta}_{W}: W \rightarrow B_{\zeta_{W}}^{q-1}$ be a $(q-1)$-smoothing of $B_{\zeta_{W}}^{q-1}$. Let $i: M \rightarrow W$ be the inclusion. Then the map

$$
\psi:=\bar{\zeta}_{W} \circ i: M \rightarrow B_{\zeta_{W}}^{q-1}
$$

defines a $\left(B_{\zeta_{W}}^{q-1}, \eta_{\zeta_{W}}^{q-1}\right)$-structure on $M$ which is compatible with $\zeta=S \varphi$ since $\left.J\right|_{\partial W}=\varphi$. Since the smooth manifold $W$ admits a handle decomposition with handles only of index $(q+1)$ or less, by turning such a decomposition upside down we conclude that $W$ has a handle decomposition starting from $M$ and adding handles of dimension $(q+1)$ and higher. It follows that $i: M \rightarrow W$ is a $q$-equivalence and hence the map $\xi: M \rightarrow B_{\zeta_{W}}^{q-1}$ is a $q$-equivalence. Since $\left(B_{\zeta_{W}}^{q-1}, \eta_{\zeta_{W}}^{q-1}\right)$ is the complex normal $(q-1)$-type of $W$, the map $\eta_{\zeta_{W}}^{q-1}: B_{\zeta_{W}}^{q-1} \rightarrow B U$ is a $q$-coequivalence. It follows that $\left(B_{\zeta_{W}}^{q-1}, \eta_{\zeta_{W}}^{q-1}\right)$ is a model for the complex normal $(q-1)$ type of $(M, \zeta)$ and so we identify $\left(B_{\zeta}^{q-1}, \eta_{\zeta}^{q-1}\right)=\left(B_{\zeta_{W}}^{q-1}, \eta_{\zeta_{W}}^{q-1}\right)$. By construction the map $\psi: M \rightarrow B_{\zeta}^{q-1}$ is a complex normal $(q-1)$-smoothing and $\left(W, \bar{\zeta}_{W}\right)$ is a $\left(B_{\zeta}^{q-1}, \eta_{\zeta}^{q-1}\right)$-null bordism of $(M, \psi)$. It follows that $[M, \psi]=0 \in \Omega_{2 q+1}\left(B_{\zeta}^{q-1} ; \eta_{\zeta}^{q-1}\right)$. Now by Lemma 2.12 , $[M, \bar{\zeta}]=0 \in \Omega_{2 q+1}\left(B_{\zeta}^{q-1}: \eta_{\zeta}^{q-1}\right)$ for any complex normal $(q-1)$-smoothing $\bar{\zeta}: M \rightarrow B_{\zeta}^{q-1}$.

$(2) \Rightarrow(3)$ : Take $(B, \eta)=\left(B_{\zeta}^{q-1}, \eta_{\zeta}^{q-1}\right)$.

$(3) \Rightarrow(1)$ : Let $\left(W, \bar{\zeta}_{W}\right)$ be a $B$-nullbordism of $(M, \bar{\zeta})$. Using surgery below the middle dimension as in Proposition 2.6, we may assume that $\bar{\zeta}_{W}: W \rightarrow B$ is a $(q+1)$-equivalence and in particular there are isomorphisms of fundmental groups $\pi=\pi_{1}(M) \cong \pi_{1}(B) \cong \pi_{1}(W)$. If $i: M \rightarrow W$ denotes, the inclusion, the commutative diagram

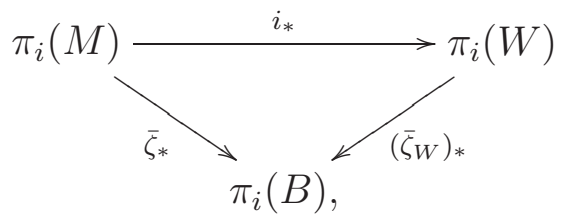


and the facts that $\bar{\zeta}: M \rightarrow B$ is a $q$-equivalence and $\bar{\zeta}_{W}: W \rightarrow B$ is a $(q+1)$-equivalence show that the inclusion $i: M \rightarrow W$ is a $q$-equivalence. By Theorem 2.18, it follows that $W$ is diffeomorphic to a manifold obtained from $M$ by attaching handles in dimension $(q+1)$ and higher.

Turning the above handle decomposition upside down, we see that $W$ has a handle decomposition consisting of $k$-handles with $k \leq q+1$. Moreover, the $(B, \eta)$-strucure on $W$ defines a stable complex structure $\zeta_{W}$ on $W$. Applying Lemma 2.16 to the handlebody decomposition of $W$ we deduce that $W$ admits an almost complex structure $J$ such that $S J=\zeta_{W}$. The almost complex structure $J$ induces some almost contact structure $\left.J\right|_{\partial W}$ on $M$ such that $\left.S J\right|_{\partial W}=S \varphi$. It follows that $\varphi=\left.J\right|_{\partial W}+\varphi_{0}$ where $\varphi_{0} \in \pi_{2 q+1}(S O(2 q+1) / U(q))$ is a stably trivial almost contact structure on $S^{2 q+1}$. By Lemma 2.17 the almost contact manifold $\left(S^{2 q+1}, \varphi_{0}\right)$ admits a Stein filling $\left(W_{0}, \sigma_{0}\right)$ and in particular an almost complex filling $\left(W_{0}, J_{0}\right)$. It follows that the boundary connected sum $\left(W \downarrow W_{0} ; J \downarrow J_{0}\right)$ is an almost complex filling of $\left.J\right|_{\partial W}+\varphi_{0}=\varphi$.

The above result admits a 'relative' version, where consider bordisms between two smoothings:

Lemma 2.20 (Stable surgery Lemma). Let $\left(W, \bar{\zeta}_{W} ; M_{0}, M_{1}\right)$ be a $(B, \eta)$-bordism between normal $(q-1)$-smoothings $\left(M_{0}, \bar{\zeta}_{0}\right)$ and $\left(M_{1}, \bar{\zeta}_{1}\right)$ of dimension $2 q+1 \geq 5$. Then for $j=0,1$ the bordism $W$ admits a handlebody decomposition relative to $M_{j}$ consisting of handles of index $k \leq q+1$.

Proof. Let $i_{j}: M_{j} \rightarrow W, j=0,1$ denote the inclusion maps. Using surgery below the middle dimension as in Proposition 2.6, we may assume that $\bar{\zeta}_{W}: W \rightarrow B$ is a $(q+1)$-equivalence. Now consider the following commutative diagram

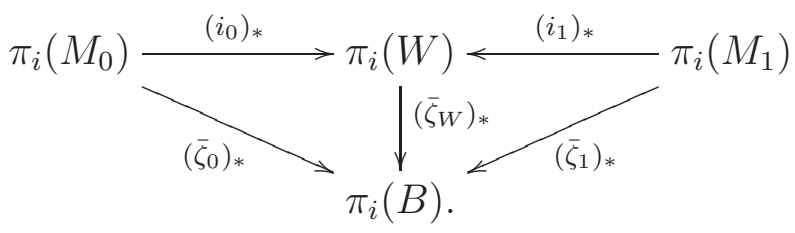

Since the maps $\bar{\zeta}_{i}: M_{i} \rightarrow B$ are $q$-equivalences and $\bar{\zeta}_{W}: W \rightarrow B$ is a $(q+1)$-equivalence, it follows that each inclusion $i_{j}: M_{j} \rightarrow W$ is a $q$-equivalence. By Theorem 2.18, $W$ admits a handlebody decomposition relative to $M_{j+1}$ consisting of handles of index $k^{\prime} \geq q+1$. If we turn this handbody decomposition upside down we obtain a handlebody decomposition of $W$ relative to $M_{j}$ consisting of handles of index $k \leq q+1$.

We next give the unstable version of the previous lemma:

Lemma 2.21 (Unstable surgery Lemma). Let $\left(M_{0}, \varphi_{0}\right)$ and $\left(M_{1}, \varphi_{1}\right)$ be almost contact manifolds of dimension $2 q+1 \geq 5$ with associated stable complex structures $\zeta_{0}$ and $\zeta_{1}$. Suppose for $i=0,1$, that $\bar{\zeta}_{i}: M_{i} \rightarrow B$, are $\zeta_{i}$-compatible normal $(q-1)$-smoothings in a stable complex bundle $(B, \eta)$ which are $(B, \eta)$-bordant. Then there is an almost complex bordism $\left(W, J ;\left(M_{0}, \varphi_{0}\right),\left(M_{1}, \varphi_{1}\right)\right)$ between $\left(M_{0}, \varphi_{0}\right)$ and $\left(M_{1}, \varphi_{1}\right)$ such that for $j=0,1$ the manifold $W$ admits a handlebody decomposition relative to $M_{j}$ consisting of handles of index $k \leq q+1$.

Proof. Let us give the proof for $j=0$, the proof for $j=1$ is similar. By Lemmas 2.16 and 2.20 there is an almost complex bordism $\left(W, J ;\left(M_{0}, \varphi_{0}\right),\left(M_{1}, \varphi_{1}\right)\right)$ where $W$ is obtained from $M_{0}$ by attaching handles of index $(q+1)$ or less and where the almost contact structure 
$\varphi_{1}^{\prime}$ satisfies $S \varphi_{1}^{\prime}=S \varphi_{1}$. It follows that $\varphi_{1}=\varphi_{1}^{\prime}+\varphi_{0}$ where $\varphi_{0} \in \pi_{2 q+1}(S O(2 q+1) / U(q))$ is a stably trivial almost contact structure on $S^{2 q+1}$. By Lemma 2.17 the almost contact manifold $\left(S^{2 q+1}, \varphi_{0}\right)$ admits an almost complex filling $\left(W_{0}, J_{0}\right)$ with handles of index $\leq q+1$. Taking the boundary connected sum of $W$ and $W_{0}$ at the $M_{1}$ boundary component of $W$ we obtain an almost complex bordism $\left(W \downarrow W_{0}, J \downarrow J_{0} ;\left(M_{0}, \varphi_{0}\right),\left(M_{1}, \varphi_{1}\right)\right)$ where $W \downarrow W_{1}$ has a handlebody decomposition relative to $M_{0}$ consisting of handles of index $(q+1)$ or less.

2.5. Complex normal $k$-types. In this subsection, we identify the complex normal $k$-type $\left(B_{\zeta}^{k}, \eta_{\zeta}^{k}\right)$, of a general stably complex manifold $(X, \zeta)$ under certain assumptions for $k=1$ and $k=2$. These computations will play crucial roles in our applications (cf. Section 4 ). We shall use the following notation. Since we do not distinguish between stable complex bundles and their classifying maps, we shall write $f \oplus g: X \times Y \rightarrow B U$ for the exterior Whitney sum of stable complex bundles classified by maps $f: X \rightarrow B U$ and $g: Y \rightarrow B U$. Also, we let $\pi_{S U}: B S U \rightarrow B U$ be the map of classifying spaces induced by the inclusion $S U \subset U$.

Lemma 2.22. Let $(X, \zeta)$ be a stably complex manifold with $\pi=\pi_{1}(X)$.

(1) If $\zeta_{*}: \pi_{2}(X) \rightarrow \pi_{2}(B U)$ is onto then

$$
\left(B_{\zeta}^{1}, \eta_{\zeta}^{1}\right)=\left(K(\pi, 1) \times B U, \operatorname{pr}_{B U}\right) .
$$

(2) If $c_{1}(\zeta)=0 \in H^{2}(X)$ then

$$
\left(B_{\zeta}^{1}, \eta_{\zeta}^{1}\right)=\left(K(\pi, 1) \times B S U, \pi_{S U} \circ \operatorname{pr}_{B U}\right) .
$$

Proof. Both $\mathrm{pr}_{B U}$ and $\pi_{S U} \circ \mathrm{pr}_{B U}$ are 2-coequivalences. Thus, from the defining properties of the second Postnikov approximation of $\zeta: X \rightarrow B U$, it suffices to find maps $\bar{\zeta}: X \rightarrow B_{\zeta}^{1}$ which are 2-equivalences and which factor $\zeta$ over $\eta_{\zeta}^{1}$.

(11) Let $u: X \rightarrow K(\pi, 1)$ classify the universal covering of $X$ and define $\bar{\zeta}$ by

$$
\bar{\zeta}:=(u \times \zeta): X \rightarrow K(\pi, 1) \times B U .
$$

The assumption that $\zeta_{*}$ in onto on $\pi_{2}$ ensures that $\zeta$ is a 2-equivalence and clearly $\operatorname{pr}_{B U} \circ \bar{\zeta}=\zeta$.

(2) Since $c_{1}(\zeta)=0$, there is a lift of $\zeta$ to $\zeta^{\prime}: X \rightarrow B S U$. Define $\bar{\zeta}$ by

$$
\bar{\zeta}:=\left(u \times \zeta^{\prime}\right): X \rightarrow K(\pi, 1) \times B S U .
$$

Since $\pi_{2}(B S U)=0, \zeta$ is a 2-equivalence and clearly $\pi_{S U} \circ \operatorname{pr}_{B U} \circ \bar{\zeta}=\zeta$.

Now we consider the complex normal 2-type of $(X, \zeta)$. Let $p_{2}: X \rightarrow P_{2}(X)$ denote a 3-equivalence from $X$ to its second Postnikov stage, $P_{2}(X)$.

Lemma 2.23. Let $(X, \zeta)$ be a stably complex manifold and let $\gamma_{\zeta}$ by the unique complex line bundle over $P_{2}(X)$ such that $c_{1}\left(p_{2}^{*}\left(\gamma_{\zeta}\right)\right)=-c_{1}(\zeta)$. Then

$$
\left(B_{\zeta}^{2}, \eta_{\zeta}^{2}\right)=\left(P_{2}(X) \times B S U, \gamma_{\zeta} \oplus \pi_{S U}\right) .
$$

Proof. By definition, the map on second cohomology induced by $p_{2}$ is an isomorphism: $p_{2}^{*}: H^{2}\left(P_{2}(X)\right) \cong H^{2}(X)$. Hence there is a (unique isomorphism class of) line bundle $\gamma_{\zeta}$ over $P_{2}(X)$ such that $p_{2}^{*}\left(\gamma_{X}\right)=-c_{1}(\zeta)$. The stable complex bundle $\xi:=\zeta \oplus p_{2}^{*}\left(\gamma_{\zeta}\right)$ satisfies

$$
c_{1}(\xi)=c_{1}(\zeta)-c_{1}(\zeta)=0 \in H^{2}(X),
$$

and so $\xi$ admits an $S U$-structure classified by a map $\xi^{\prime}: X \rightarrow B S U$. We define $\bar{\zeta}$ by

$$
\bar{\zeta}:=\left(p_{2} \times \xi^{\prime}\right): X \rightarrow P_{2}(X) \times B S U .
$$


Since $B S U$ is 3-connected and $\pi_{3}\left(P_{2}(X)\right)=0, \bar{\zeta}$ is a 3-equivalence. By construction we have $\left(\gamma_{\zeta} \oplus \pi_{S U}\right) \circ \bar{\zeta}=\zeta$ and clearly $\gamma_{\zeta} \oplus \pi_{S U}$ is a 3 -coequivalence. It follows that $\left(B_{\zeta}^{2}, \eta_{\zeta}^{2}\right)$ is the complex normal 2-type of $(X, \zeta)$.

\section{Contact structures and COMPlex normal Bordism}

After recalling the necessary definitions and the statement of Eliashberg's $h$-principle, we state our main surgery theorems, Theorems 3.7 and 3.8. The proofs of these theorems rest on the discussion presented in Section 2.4.

3.1. Symplectic fillability and contact surgery. Recall that a symplectic manifold $(W, \omega)$ is a $(2 q+2)$-dimensional manifold $W$ with a closed 2 -form $\omega$ such that $\omega^{q+1} \neq 0$ at every point in $W$. In particular, a symplectic manifold carries a canonical orientation. Recall, furthermore, that a cooriented, codimension-1 distribution $\xi$ on a $(2 q+1)$-manifold $M$ is a contact structure if there is a 1 -form $\alpha$ such that $\operatorname{ker}(\alpha)=\xi$ and

$$
\alpha \wedge(d \alpha)^{q} \neq 0 \text {. }
$$

Note that this then also determines an orientation of $M$. Two contact manifolds $\left(M_{0}, \xi_{0}\right)$ and $\left(M_{1}, \xi_{1}\right)$ are contactomorphic if there is a diffeomorphism $\phi: M_{0} \rightarrow M_{1}$ such that

$$
\phi_{*}\left(\xi_{0}\right)=\xi_{1} .
$$

We now recall the various notions of fillability for contact structures.

Definition 3.1 (Strongly symplectically fillable and exactly fillable). A contact manifold $(M, \xi)$ is called strongly symplectically fillable if it bounds a compact symplectic manifold $(W, \omega)$ and there is an outward pointing vector field $V$ near $\partial X$ such that the Lie derivative satisfies $L_{V} \omega=\omega$, and $\lambda=\iota_{V} \omega$ is a defining 1 -form for $\xi$. If the symplectic form $\omega$ is exact then we say that $(M, \xi)$ is exactly fillable.

A further specialisation of the notion of fillability is that of Stein fillability. Recall that a Stein domain is a compact, complex manifold $(W, J)$ with boundary that admits a function $\phi: W \rightarrow[0,1]$ so that $\omega=-d d^{\mathbb{C}} \phi$ is a symplectic form and $\phi^{-1}(1)=\partial W$ is a regular level.

Definition 3.2 (Stein fillable). A contact manifold $(M, \xi)$ is called Stein fillable if it bounds a Stein domain $(W, J)$ such that $\xi=J(T M) \cap T M$.

These notions of fillability fit into the following sequence of inclusions of contactomorphism classes of contact manifolds:

$$
\{\text { Stein fillable }\} \subseteq\{\text { exactly fillable }\} \subseteq\{\text { strongly fillable }\} .
$$

A $k$-sphere $S^{k} \subset M$ in an contact manifold $\left(M^{2 q+1}, \xi\right)$ is called isotropic if $T S^{k} \subset \xi$. Surgery on an isotropic sphere $S^{k}$ can be performed in a way that is compatible with the contact structure. If $k \leq q$ and $2 q+1 \geq 5$ then any embedded sphere can be realised by an isotropic sphere and such surgeries can be realised by the attachment of a symplectic or "Weinstein" $(k+1)$-handle $h_{k+1}:=D^{k+1} \times D^{2 q+1-k}$, provided that the associated almost complex structure on the product manifold $M^{2 q+1} \times[0,1]$ extends over the trace $\left(M^{2 q+1} \times[0,1]\right) \cup h_{k+1}$ of the surgery (cf. [El1, CE]) . Furthermore, the symplectic nature of the handle attachment shows that the symplectic fillability of a contact structure is preserved under such contact surgeries. In addition, Eliashberg showed that when attaching a Weinstein handle to a Stein manifold, the Stein structure also extends. (For more details concerning contact surgery and Weinstein handles we refer the reader to [CE] or [Ge4] or [We].) 
Theorem 3.3. Let $\left(M^{2 q+1}, \xi\right)$ be a contact manifold of dimension $2 q+1 \geq 5$ with associated almost contact structure $\varphi$. Suppose that $k \leq q$ and that $\left(M^{\prime}, \varphi^{\prime}\right)$ is obtatined from $(M, \varphi)$ via a $k$-dimensional almost complex surgery with trace $(M \times I) \cup h_{k+1}$ as in Defintion 2.14. Then $M^{\prime}$ admits a contact structure $\xi^{\prime}$. If is $\left(M^{2 q+1}, \xi\right)$ symplectically or exactly fillable, then so is $\left(M^{\prime}, \xi^{\prime}\right)$. Moreover, if $(W, J)$ is a Stein filling of $\left(M^{2 q+1}, \xi\right)$ then there is $J^{\prime}$ on $W \cup h_{k+1}$ such that it is a Stein filling of $\left(M^{\prime}, \xi^{\prime}\right)$.

Remark 3.4. Although [Ge4, Theorem 6.3.1] is not stated explicitly for exact fillability, the proof also holds in the case of exact fillability, since attaching Weinstein handles does not affect the exactness of the symplectic form on the filling.

Applying Theorem 3.3 inductively over a handle decomposition, one obtains the following (cf. [CE], Theorem 8.15):

Corollary 3.5 (Eliashberg's $h$-principle). Let $(W, J)$ be a compact $(2 q+2)$-dimensional almost complex manifold with handles only in dimensions $q+1$ or less. Then $J$ is homotopic to an almost complex structure $\tilde{J}$ so that $(W, \tilde{J})$ is a Stein filling of $M=\partial W$ and in particular, $M$ is Stein fillable.

3.2. Surgery theorems. In this subsection we state our main theorems concerning Stein fillings and contact surgery. The results will be mainly translations of the surgery theoretic results from Section 2.4. We begin with the result corresponding to Lemma 2.17.

Lemma 3.6. Suppose that the almost contact manifold $(M, \varphi)$ can be realised as a contact structure $\xi$. Then every homotopy class of almost contact structure which is stably equivalent to $\varphi$ admits a contact structure obtained from $\xi$ by connected sum with a Stein fillable contact structure on $S^{2 q+1}$.

Proof. The proof is a simple combination of the proof of Lemma 2.17 and Corollary 3.5 . the almost contact structures found on $S^{2 q+1}$ in the proof of Lemma 2.17 are Stein fillable contact structures, and the boundary connect sum of two Stein fillings is a Stein filling.

Using the notation and terminology of Section 2, we obtain the following bordism characterisation of Stein fillability, proving (an expanded version of) Theorem 1.2.

Theorem 3.7 (Filling Theorem). Let $(M, \varphi)$ be a closed almost contact $(2 q+1)$-manifold with induced stable complex structure $\zeta$ and complex normal $(q-1)$-type $\left(B_{\zeta}^{q-1}, \eta_{\zeta}^{q-1}\right)$. If $q \geq 2$, then the following are equivalent:

(1) $(M, \varphi)$ admits a Stein-fillable contact structure;

(2) for any $\zeta$-compatible normal $(q-1)$-smoothing $\bar{\zeta}: M \rightarrow B_{\zeta}^{q-1}$, we have

$$
[M, \bar{\zeta}]=0 \in \Omega_{2 q+1}\left(B_{\zeta}^{q-1} ; \eta_{\zeta}^{q-1}\right)
$$

(3) for some stable complex bundle $(B, \eta)$ and some $\zeta$-compatible normal (q-1)-smoothing $\bar{\zeta}: M \rightarrow B$, we have

$$
[M, \bar{\zeta}]=0 \in \Omega_{2 q+1}(B ; \eta)
$$

Proof. For stable almost contact structures this is just a combination of Lemma 2.19 and Eliashberg's $h$-principle (cf. Corollary 3.5). Lemma 3.6 then implies that any almost contact structure in a given stable class can be realised as a Stein fillable contact structure, as soon as one can. 
Similar arguments provide

Theorem 3.8 (Surgery Theorem). Let $\left(M_{0}, \varphi_{0}\right)$ and $\left(M_{1}, \varphi_{1}\right)$ be almost contact manifolds of dimension $2 q+1 \geq 5$ with associated stable complex structures $\zeta_{0}$ and $\zeta_{1}$. Suppose for $i=0,1$, that $\bar{\zeta}_{i}: M_{i} \rightarrow B$ are $\zeta_{i}$-compatible normal $(q-1)$-smoothings in a stable complex bundle $(B, \eta)$ such that

$$
\left[M_{0}, \bar{\zeta}_{0}\right]=\left[M_{1}, \bar{\zeta}_{1}\right] \in \Omega_{2 q+1}(B ; \eta)
$$

Then $\left(M_{0}, \varphi_{0}\right)$ admits a contact structure if and only if $\left(M_{1}, \varphi_{1}\right)$ does. Moreover, $\left(M_{0}, \varphi_{0}\right)$ admits a fillable contact structure in any sense (cf. display (3) above) if and only if $\left(M_{1}, \varphi_{1}\right)$ does.

Proof. By Lemma 2.20, there is a $(B, \eta)$-bordism $\left(W, \bar{\zeta}_{W}\right)$ between $\left(M_{0}, \bar{\zeta}_{0}\right)$ and $\left(M_{1}, \bar{\zeta}_{1}\right)$ such that $\left(W, \bar{\zeta}_{W}\right)$ is obtained from $\left(M_{i}, \bar{\zeta}_{i}\right) \times[0,1], i=0,1$, by attaching $k$-handles, $k \leq q+1$, over which the almost complex structure extends. The result now follows from Theorem 3.3 above. This then gives contact structures in the desired stable class of almost contact structures. However, by Lemma 3.6 one can then realise all almost contact structures via connected sum with certain contact structures on spheres. As all these contact structures are Stein fillable, this does not affect the fillability of the contact structures.

Remark 3.9. The idea of constructing contact structures via surgery techniques is not new, and Geiges and Thomas, in particular, have employed such methods to prove the existence of contact structures under various topological assumptions. Indeed, using the explicit description of normal 1-types given in Lemma 2.22, one can deduce the Bordism Theorem of [Ge3] as a special case of Theorem 3.8. The main benefit of Theorem 3.8 is that it provides a unified approach to this point of view without making any assumptions on the almost contact structures involved.

In the following sections we will use the Filling Theorem above to produce Stein fillable contact structures and obstructions to Stein fillability. The Surgery Theorem, on the other hand, is useful for finding contact structures on manifolds which cannot carry Stein fillable structures as we now explain. Let $\beta$ denote a class of contact structures which is closed under Weinstein handle attachment and which includes Stein fillable contact structures; for example $\beta$ could be the class of symplectically fillable contact structures. We define

$$
\Omega_{2 q+1}^{\beta}(B ; \eta) \subset \Omega_{2 q+1}(B ; \eta)
$$

to be the set of bordism classes with representatives $\bar{\zeta}: N \rightarrow B$ such that $\bar{\zeta}$ is $\zeta$-compatible and such that $(N, \zeta)$ admits a contact structure $\xi$ in the class $\beta$. We emphasise that here we make no connectivity assumption on the $\operatorname{map} \bar{\zeta}: N \rightarrow B$.

Corollary 3.10. Let $(M, \varphi)$ be an almost contact $(2 q+1)$-manifold with associated stable complex structure $\zeta$ and let $(B, \eta)$ be a stable complex bundle. If $q \geq 2$, the map $\bar{\zeta}: M \rightarrow B$ is a $\zeta$-compatible normal $(q-1)$-smoothing and

$$
[M, \bar{\zeta}] \in \Omega_{2 q+1}^{\beta}(B ; \eta)
$$

then $(M, \zeta)$ admits a contact structure in the class $\beta$.

Proof. By assumption, there is a contact manifold $(N, \xi)$ with associated stable complex structure $\zeta_{N}$ and with a $\zeta_{N}$-compatibe $(B, \eta)$-structure $\bar{\zeta}_{N}: N \rightarrow B$ such that $\left[N, \bar{\zeta}_{N}\right]=$ $[M, \bar{\zeta}] \in \Omega_{2 q+1}(B ; \eta)$. By Proposition 2.6 , we may perform $(B, \eta)$-surgeries of dimension $q$ or 
less on $\bar{\zeta}: N \rightarrow B$ to obtain a $(q-1)$-smoothing $\bar{\zeta}_{N^{\prime}}: N^{\prime} \rightarrow B$, with induced stable complex structure $\zeta^{\prime}$ say. By Lemmas [2.16] and 2.17 and Theorem [3.3, $\left(N^{\prime}, \varphi^{\prime}\right)$ admits a contact structure $\xi^{\prime}$ in the class $\beta$ and with associated almost contact structure $\varphi^{\prime}$ which stabilises to $\zeta^{\prime}$. Applying Theorem 3.8 to $\left(N^{\prime}, \varphi^{\prime}\right)$ and $(M, \varphi)$, we deduce that $(M, \varphi)$ admits a contact structure in the class $\beta$.

The line of reasoning from the proof of Corollary 3.10 was pursued in [BCS1] for $\beta$ the class of all contact structures. There contact structures on manifolds of the form $M \times S^{2}$ with $M$ contact were shown to exist by finding Stein cobordisms from $M \times T^{2}$ and applying a result of Bourgeois [Bou which provides a contact structure for this latter manifold once $M$ is contact. More generally, Corollary 3.10 gives a framework for approaching the symplectic version of the Stein Realisation Problem 1.1:

Problem 3.11 (Symplectic Relaization Problem). Determine which almost contact structures on a given manifold can be realised by strongly/exactly fillable contact structures. Does the answer depend on whether one considers strong or exact fillings?

\section{Simply CONNECTED 7-MANifOLDS}

As an application of the methods developed in Section 3, we now give a proof of Theorem 1.3. The proof will show that the Stein fillability obstruction of Theorem 3.7 vanishes by showing that the relevant bordism group is itself trivial.

Before turning to the computation of the bordism group, however, we show that every 7-manifold considered in Theorem 1.3 admits an almost contact structure.

To start the argument, recall that a manifold $M$ admits a spin ${ }^{c}$ structure, that is, a lift of the structure group of $T M$ from $S O(n)$ to the group $\operatorname{Spin}^{\mathbb{C}}(n)$, if and only if the second Stiefel-Whitney class $w_{2}(M) \in H^{2}\left(M ; \mathbb{Z}_{2}\right)$ admits an integral lift. (The Lie group $\operatorname{Spin}^{\mathbb{C}}(n)$ can be defined as the extension of $S O(n)$ by $S^{1}$ with the property that $\operatorname{Spin}^{\mathbb{C}}(n) \rightarrow S O(n)$ is the unique nontrivial principal $S^{1}$-bundle over $S O(n)$.) Since each manifold $M$ in Theorem 1.3 is simply connected and has torsion free $\pi_{2}(M) \cong H_{2}(M)$, the mod 2 reduction map $H^{2}(M) \rightarrow H^{2}\left(M ; \mathbb{Z}_{2}\right)$ is onto, and so $M$ admits a spin ${ }^{c}$ structure.

Notice that $U(n) \subset S O(2 n)$, and since any $S^{1}$-bundle over $U(n)$ is trivial (by the fact that $\left.H^{2}(U(n))=0\right)$, we have that the restriction of the bundle $\operatorname{Spin}^{\mathbb{C}}(2 n) \rightarrow S O(2 n)$ over $U(n)$ is trivial. Consequently $\operatorname{Spin}^{\mathbb{C}}(2 n)$ contains $U(n) \times S^{1}$, so in particular $U(n)$ embeds into $\operatorname{Spin}^{\mathbb{C}}(2 n)$. This embedding provides a homomorphism of topological groups $U \rightarrow \operatorname{Spin}^{\mathbb{C}}$. Similarly, $S U(n)$ embeds into $S O(2 n)$, and since $S U(n)$ is simply connected, this embedding lifts to an embedding $S U(n) \rightarrow \operatorname{Spin}(2 n)$ (recall that $\operatorname{Spin}(2 n) \rightarrow S O(2 n)$ is the nontrivial double cover of $S O(2 n)$ ). This construction then provides a homomorphism of topological groups $S U \rightarrow$ Spin.

We first show that every $\operatorname{spin}^{c}$ structure on a 7 -manifold is induced by an almost contact structure.

Lemma 4.1. A compact oriented 7-manifold $X$ admits an almost contact structure if and only if it admits a spin structure. Moreover, any spin ${ }^{c}$ structure on $X$ is induced from some almost contact structure on $X$.

Proof. By Lemma 2.17 (1), any stable complex structure $\zeta$ on $X$ can be destabilised to an almost contact structure $\varphi$. Hence it is enough to show that $X$ admits a stable complex structure if and only if $X$ admits a stable $\operatorname{spin}^{c}$ structure, that is, a map into BSpin ${ }^{\mathbb{C}}$ covering 
the map $X \rightarrow B S O$ given by the stable tangent bundle. Since a stable complex structure on $X$ induces a $\operatorname{spin}^{c}$ structure on $X$, we only need to show that any stable $\operatorname{spin}^{c}$ structure on $X$ can be lifted to a stable complex structure.

The homomorphism of topological groups $U \rightarrow S p i n^{\mathbb{C}}$ induces a map of classifying spaces which gives a fibre bundle

$$
\operatorname{Spin}^{\mathbb{C}} / U \stackrel{i}{\rightarrow} B U \rightarrow B \operatorname{Spin}^{\mathbb{C}} .
$$

By Bott periodicity the quotient $\operatorname{Spin}^{\mathbb{C}} / U$ is 5 -connected and $\pi_{6}\left(\operatorname{Spin}^{\mathbb{C}} / U\right) \cong \mathbb{Z}$. Suppose that $\theta: X \rightarrow B \operatorname{Spin}^{\mathbb{C}}$ is a $\operatorname{spin}^{c}$ structure on $X$. We must show that the following lifting problem has a solution:

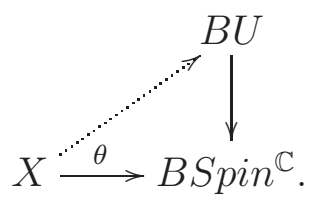

Since $\operatorname{Spin}^{\mathbb{C}} / U$ is 5 -connected, the primary obstruction to lifting $\theta$ is a cohomology class $\theta^{*}(\alpha) \in H^{7}(X)$, where we have identified $\pi_{6}\left(\operatorname{Spin}^{\mathbb{C}} / U\right)$ with $\mathbb{Z}$ and the universal obstruction class $\alpha \in H^{7}\left(\right.$ BSpin $\left.^{\mathbb{C}}\right)$ is defined below. We shall show that $2 \alpha=0$. Since $H^{7}(X)$ is torsion free, it follows that $\theta^{*}(\alpha)=0$ and hence $\theta$ lifts to a stable complex structure on $X$.

It remains to define $\alpha$ and to prove that $2 \alpha=0$. Let $x \in \pi_{6}\left(\operatorname{Spin}^{\mathbb{C}} / U\right) \cong \mathbb{Z}$ be a generator. Since $\operatorname{Spin}^{\mathbb{C}} / U$ is 5 -connected, there is a generator $\widehat{x} \in H^{6}\left(\operatorname{Spin}^{\mathbb{C}} / U\right)$ such that $\langle\widehat{x}, \rho(x)\rangle=1$ where $\rho: \pi_{6}\left(\operatorname{Spin}^{\mathbb{C}} / U\right) \rightarrow H_{6}\left(\operatorname{Spin}^{\mathbb{C}} / U\right)$ is the Hurewicz homomorphism. The class $\widehat{x}$ is transgressive in the Leray-Serre cohomology spectral sequence of the fibration (44), and we define

$$
\alpha:=\tau(\widehat{x}) \in H^{7}\left(\operatorname{BSpin}^{\mathbb{C}}\right),
$$

where $\tau: H^{6}\left(\operatorname{Spin}^{\mathbb{C}} / U\right) \rightarrow H^{7}\left(B \operatorname{Spin}^{\mathbb{C}}\right)$ is the transgression homomorphism. Since the kernel of $\tau$ is the image of the homomoprhism $i^{*}: H^{6}(B U) \rightarrow H^{6}\left(\operatorname{Spin}^{\mathbb{C}} / U\right) \cong \mathbb{Z}$, it suffices to show that the image of $i^{*}$ is the subgroup of index two. Now $H^{*}(B U)=\mathbb{Z}\left[c_{1}, c_{2}, c_{3}, \ldots\right]$ is the polynomial algebra on the Chern classes and the composition $S^{6} \stackrel{x}{\rightarrow} \operatorname{Spin}^{\mathbb{C}} / U \stackrel{i}{\rightarrow} B U$ determines the stable complex vector bundle $x^{*} i^{*}(E U)$ over $S^{6}$ where $E U \rightarrow B U$ is the universal bundle. By [Hu, Chapter7, Corollary 9.9], every complex bundle $E$ over $S^{6}$ is such that $c_{3}(E) \in 2 \cdot H^{6}\left(S^{6}\right)$ and moreover there is a complex bundle $E_{0}$ over $S^{6}$ where $c_{3}\left(E_{0}\right)$ is twice a generator of $H^{6}\left(S^{6}\right)$. It follows that $i^{*}\left(H^{6}(B U)\right)=2 \cdot H^{6}\left(\operatorname{Spin}^{\mathbb{C}} / U\right)$ and the lemma follows.

We now reduce the proof of Theorem 1.3 to the calculation of certain bordism groups. Let $\varphi$ be an almost contact structure on $M$, with associated stable complex structure $\zeta$, let $H=H_{2}(M)$ and let $\gamma$ be the complex line bundle over the Eilenberg-MacLane space $K(H, 2)$ with $c_{1}(\gamma)=-c_{1}(\zeta) \in H^{2}(K(H, 2) ; \mathbb{Z}) \cong H^{2}(M ; \mathbb{Z})$. By Lemma 2.23, the complex normal 2-type of the stably complex manifold $(M, \zeta)$ is

$$
\left(B_{\zeta}^{2} ; \eta_{\zeta}^{2}\right)=\left(K(H, 2) \times B S U ; \gamma \oplus \pi_{S U}\right),
$$

where $\pi_{S U}: B S U \rightarrow B U$ is the map induced by the inclusion $S U \rightarrow U$ and $\oplus$ denotes the exterior Whitney sum of complex bundles: for further details, see Section 2.5. It follows that there is an isomorphism of bordism groups

$$
\Omega_{7}\left(B_{\zeta}^{2} ; \eta_{\zeta}^{2}\right) \cong \Omega_{7}^{S U}(K(H, 2) ; \gamma)
$$


where the latter group is a certain $\gamma$-twisted $S U$-bordism group of $K(H, 2)$. This is the bordism group of triples $(N, f, \alpha)$ where $N$ is a closed smooth manifold, $f: N \rightarrow K(H, 2)$ is a map and $\alpha$ is an $S U$ structure on the Whitney sum of $f^{*}(\gamma)$ and the stable normal bundle of $N$.

The remainder of this subsection gives the proof of the following proposition.

Proposition 4.2. For any finitely generated free abelian group $H$ and for any complex line bundle $\gamma$ over $K(H, 2)$, we have $\Omega_{7}^{S U}(K(H, 2) ; \gamma)=0$.

We shall need the following result on $S U$-bordism groups.

Lemma 4.3. If $k$ is not divisible by 4 then $\Omega_{2 k+1}^{S U}=0$.

Proof. By [Stn, p. 117], we know that $\Omega_{*}^{U}=0$ for any odd dimension $*=2 k+1$. Also by [Stn, p. 238], the kernel of the forgetful homomorphism $\Omega_{*}^{S U} \rightarrow \Omega_{*}^{U}$ is the torsion subgroup of $\Omega_{*}^{S U}$. But by [Stn, p. 248], the torsion subgroup of $\Omega_{2 k+1}^{S U}$ vanishes if $k$ is not divisible by 4 , concluding the proof.

For the case $H=0$ in Proposition 4.2, by Lemma 4.3 implies that $\Omega_{7}^{S U}=0$ as required. Hence we assume that $H$ is not the zero group. We wish to compute the $\gamma$-twisted $S U$ bordism of $K(H, 2)$. A very similar situation is discussed in [KS, Section 6] where Kreck and Stolz compute certain twisted spin bordism groups of $K(\mathbb{Z}, 2)$. Since the Thom space of the exterior Whitney sum of bundles is homotopy equivalent to the smash product of the individual Thom spaces, the Pontrjagin-Thom construction gives an isomorphism

$$
\Omega_{*}^{S U}(K(H, 2) ; \gamma) \cong \pi_{*}\left(T\left(\gamma \oplus \pi_{S U}\right)\right) \cong \pi_{*}(T(\gamma) \wedge M S U) \cong \widetilde{\Omega}_{*}^{S U}(T(\gamma))
$$

Here $M S U$ is the Thom spectrum defined by special unitary boridsm, $T(\gamma)$ is the Thom space of the bundle $\gamma$ over $K(H, 2), \wedge$ denotes the smash product of spectra and $\widetilde{\Omega}_{*}^{S U}$ denotes reduced special unitary bordism. As a consequence of ([6), there is an Atiyah-Hirzebruch spectral sequence (AHSS),

$$
E_{p, q}^{2}=H_{p+2}\left(T(\gamma) ; \Omega_{q}^{S U}\right) \Longrightarrow \Omega_{p+q}^{S U}(K(H, 2) ; \gamma),
$$

which converges to the associated graded object of a filtration on $\Omega_{p+q}^{S U}(K(H, 2) ; \gamma)$. By the Thom isomorphism, $\widetilde{H}^{*}(T(\gamma))$ is a free module over $H^{*}(K(H, 2))$ with generator the Thom class $U \in H^{2}(T(\gamma))$ of $\gamma$. As a consequence, $H_{*}(T(\gamma))$ vanishes in odd degrees. Now, by Lemma 4.3, $\Omega_{2 k+1}^{S U}=0$ for $k=1,2,3$ and $\Omega_{1}^{S U} \cong \mathbb{Z}_{2}$ by [Stn, p. 248]. It follows that the 7-line of the $E^{2}$-page of the AHSS above has only one non-vanishing term and that is

$$
E_{6,1}^{2}=H_{8}\left(T(\gamma) ; \Omega_{1}^{S U}\right) \cong H_{6}\left(K(H, 2) ; \Omega_{1}^{S U}\right) .
$$

We claim that $E_{6,1}^{3}=0$, which proves Proposition 4.2. To see that $E_{6,1}^{3}=0$, we need to understand the following differentials in the AHSS, where we use that fact that $\Omega_{2}^{S U} \cong \mathbb{Z} / 2$ by [Stn, p. 248]:

$$
d_{8,0}^{2}: H_{10}(T(\gamma)) \rightarrow H_{8}\left(T(\gamma) ; \mathbb{Z}_{2}\right) \text { and } d_{6,1}^{2}: H_{8}\left(T(\gamma) ; \mathbb{Z}_{2}\right) \rightarrow H_{6}\left(T(\gamma) ; \mathbb{Z}_{2}\right)
$$

Since the map $S U \rightarrow$ Spin is a 6-equivalence, these differentials for $S U$-bordism will coincide with the corresponding differentials for spin bordism. The differentials in the spin case have been computed by Teichner [Te, Lemma 2.3.2]. Hence we have the following lemma. 
Lemma 4.4 ([Te, Lemma 2.3.2]). Let $\rho_{2}: H_{*}(T(\gamma)) \rightarrow H_{*}\left(T(\gamma) ; \mathbb{Z}_{2}\right)$ be the homomorphism induced by reduction mod 2 and let $\left(S q^{2}\right)^{*}: H_{*+2}\left(T(\gamma) ; \mathbb{Z}_{2}\right) \rightarrow H_{*}\left(T(\gamma) ; \mathbb{Z}_{2}\right)$ be the dual of the Steenrod squaring operation $S q^{2}: H^{*}\left(T(\gamma) ; \mathbb{Z}_{2}\right) \rightarrow H^{*+2}\left(T(\gamma) ; \mathbb{Z}_{2}\right)$. Then the differentials in (17) above are given by

$$
d_{8,0}^{2}=\left(S q^{2}\right)^{*} \circ \rho_{2} \quad \text { and } \quad d_{6,1}^{2}=\left(S q^{2}\right)^{*}
$$

The following lemma is equivalent to the claim that $E_{6,1}^{3}=0$ in the AHSS and hence completes the proof of Proposition 4.2.

Lemma 4.5. For all finitely generated free abelian groups $H$ and for all complex line bundles $\gamma$ over $K(H, 2)$ we have

$$
\operatorname{Ker}\left(d_{6,1}^{2}\right)=\operatorname{Im}\left(d_{8,0}^{2}\right)
$$

Proof. The lemma is trivial if $H=0$, so we assume that $H$ in non-zero. We give the proof by viewing the situation from the point of view of homological algebra over the field $\mathbb{Z}_{2}$. Recall that $T(\gamma)$ denotes the Thom space of $\gamma$. We define a chain complex $\left(C_{*}(H, \gamma), d\right)$ by setting

$$
C_{i}(H, \gamma):=H_{2 i+2}\left(T(\gamma) ; \mathbb{Z}_{2}\right), \text { for } i \geq 0
$$

and defining the differential $d$ by

$$
\left.d_{i+1}:=\left(S q^{2}\right)^{*}: H_{2 i+4}\left(T(\gamma) ; \mathbb{Z}_{2}\right)\right) \rightarrow H_{2 i+2}\left(T(\gamma) ; \mathbb{Z}_{2}\right)
$$

To see that the differential satisfies $d^{2}=0$, we first recall the Adem relation $S q^{2} S q^{2}=$ $S q^{3} S q^{1}$, which entails that $S q^{2} S q^{2}=0$ on $H^{*}\left(T(\gamma) ; \mathbb{Z}_{2}\right)$ since the non-zero mod 2 cohomology groups of $T(\gamma)$ are concentrated in even degrees. It follows that $\left(S q^{2}\right)^{*}\left(S q^{2}\right)^{*}$, which is the dual of $S q^{2} S q^{2}$, vanishes.

Since the homomorphism $\rho_{2}: H_{10}(T(\gamma)) \rightarrow H_{10}\left(T(\gamma) ; \mathbb{Z}_{2}\right)$ is onto, to prove the lemma it suffices to show that the third homology group of the chain complex $\left(C_{*}(H, \gamma), d\right)$ vanishes:

$$
H_{3}\left(C_{*}(H, \gamma), d\right)=0 .
$$

In the case where $H \cong \mathbb{Z}$, it is a simple exercise using the Thom isomorphism to check that the homology of $\left(C_{*}(\mathbb{Z}, \gamma), d\right)$ is trivial if $w_{2}(\gamma) \neq 0$, and if $w_{2}(\gamma)=0$ then

$$
H_{*}\left(C_{*}(\mathbb{Z}, \gamma), d\right) \cong\left\{\begin{array}{cc}
\mathbb{Z}_{2} & *=0 \\
0 & *>0
\end{array}\right.
$$

We shall prove the general case by induction from these two cases. Let $\gamma$ be a complex line bundle over $K(H, 2)$. When $H$ has rank greater than one, let $H=H_{0} \oplus \mathbb{Z}$ with the property that $\left.c_{1}(\gamma)\right|_{H_{0}}=0$. (If $c_{1}(\gamma)=0$, then any decomposition of $H$ will do, if $c_{1}(\gamma) \neq 0$ then take $H_{0}:=\operatorname{Ker}\left(c_{1}(\gamma): H \rightarrow \mathbb{Z}\right)$.) Observe that there is a split short exact sequence $H_{0} \rightarrow H \stackrel{\pi}{\rightarrow} \mathbb{Z}$, such that $\gamma \cong \pi^{*} \gamma^{\prime}$ for the map $\pi: K(H, 2) \rightarrow K(\mathbb{Z}, 2)$ and for some complex line bundle $\gamma^{\prime}$ over $K(\mathbb{Z}, 2)$. If $\underline{\mathbb{C}}_{X}$ denotes the trivial line bundle over a space $X$, then there is an isomorphism of complex vector bundles

$$
\underline{\mathbb{C}}_{K(H, 2)} \oplus \gamma \cong \underline{\mathbb{C}}_{K\left(H_{0}, 2\right)} \oplus \gamma^{\prime}
$$

where the first $\oplus$ denotes the usual Whitney sum over $K(H, 2)$ and the second $\oplus$ the exterior Whitney sum over $K\left(H_{0}, 2\right) \times K(\mathbb{Z}, 2)=K(H, 2)$. Since the Thom space of the exterior 
Whitney sum of bundles is homotopy equivalent to the smash product of the Thom spaces of each bundle,

$$
T(\gamma) \wedge S^{2} \simeq M\left(\underline{\mathbb{C}}_{K\left(H_{0}, 2\right)}\right) \wedge T\left(\gamma^{\prime}\right) .
$$

If $x \in H^{*}\left(M\left(\underline{\mathbb{C}}_{K\left(H_{0}, 2\right)}\right) ; \mathbb{Z}_{2}\right)$ and $y \in H^{*}\left(T\left(\gamma^{\prime}\right) ; \mathbb{Z}_{2}\right)$ and $x \wedge y$ denotes their exterior cup product in $H^{*}\left(M\left(\mathbb{\mathbb { C }}_{K\left(H_{0}, 2\right)}\right) \wedge T\left(\gamma^{\prime}\right) ; \mathbb{Z}_{2}\right)$, then the Cartan formula for $S q^{2}$ gives

$$
S q^{2}(x \wedge y)=S q^{2} x \wedge y+S q^{1} x \wedge S q^{1} y+x \wedge S q^{2} y=S q^{2} x \wedge y+x \wedge S q^{2} y
$$

since $S q^{1} x$ and $S q^{1} y$ have odd degree and are thus zero. Thus there is an isomorphism of chain complexes

$$
\left(C_{*}\left(H_{0} \oplus \mathbb{Z}, \gamma\right), d\right) \cong\left(C_{*}\left(H_{0}, 0\right), d\right) \otimes\left(C_{*}\left(\mathbb{Z}, \gamma^{\prime}\right), d\right)
$$

where $\otimes$ denotes the tensor product of chain complexes. Applying the Kunneth theorem for the homology groups of a tensor product of chain complexes over $\mathbb{Z}_{2}$ inductively gives us that $H_{3}\left(C_{*}(H, \gamma), d\right)=0$ for all groups $H$ and all complex line bundles $\gamma$. This completes the proof of the lemma.

Proof of Theorem 1.3. Since a closed, oriented, simply connected manifold $M$ with torsion free $\pi_{2}(M)$ admits a $\operatorname{spin}^{c}$ structure, Lemma 4.1 implies the existence of an almost contact structure $\varphi$ on $M$. By Equation (5), Proposition 4.2 implies $\Omega_{7}\left(B_{\zeta}^{2} ; \eta_{\zeta}^{2}\right)=0$ where $\zeta=S \varphi$. It follows that for any $\zeta$-compatible normal 2-smoothing $\bar{\zeta}: M \rightarrow B_{\zeta}^{2}$, we have $[M, \bar{\zeta}]=0 \in$ $\Omega_{7}\left(B_{\zeta}^{2} ; \eta_{\zeta}^{2}\right)$. By Theorem 3.7 the almost contact manifold $(M, \varphi)$ is then Stein fillable.

\section{STEIN FILLINGS OF HOMOTOPY SPHERES}

Recall that an $n$-dimensional homotopy sphere is a closed, smooth, oriented manifold $\Sigma$ which is homotopy equivalent to $S^{n}$. The set of oriented diffeomorphism classes of homotopy $n$-spheres forms an abelian group $\Theta_{n}$ under the operation of connected sum:

$$
\Theta_{n}:=\left\{[\Sigma] \mid \Sigma \simeq S^{n}\right\}
$$

For $n \geq 5$, every homotopy $n$-sphere $\Sigma$ is homeomorphic to $S^{n}\left[\mathrm{Sm}\right.$, hence $\Theta_{n}$ may be regarded as the group of oriented diffeomorphism classes of smooth structures on the $n$ sphere.

We now recall some fundamental facts about the group $\Theta_{n}$ proved by Kervaire and Milnor. For further information, we refer the reader to [KM, Le and [Lü, 6.6]. Let $O$ denote the stable orthogonal group, $\pi_{n}^{S}$ the $n^{\text {th }}$ stable homotopy group of spheres and recall the $J_{-}$ homomorphism

$$
J_{n}: \pi_{n}(O) \rightarrow \pi_{n}^{S}
$$

Since $\pi_{n}^{S}$ is a finite group, the cokernel of $J_{n}, \operatorname{Coker}\left(J_{n}\right)$, is also finite. We state the following theorem of Kervaire and Milnor only for the case of interest to us where $n=2 q+1 \geq 5$.

Theorem 5.1 ([KM, Section 4], [KM, Theorem 6.6]). For $2 q+1 \geq 5$ the abelian group $\Theta_{2 q+1}$ lies in a short exact sequence

$$
0 \longrightarrow b P_{2 q+2} \longrightarrow \Theta_{2 q+1} \stackrel{\eta}{\longrightarrow} \operatorname{Coker}\left(J_{2 q+1}\right) \longrightarrow 0
$$

where $b P_{2 q+2}$ denotes the finite cyclic group of homotopy $(2 q+1)$-spheres which bound parallelisable manifolds.

When we move to the stable complex setting, we have the following 
Example 5.2. Every homotopy $(2 q+1)$-sphere $\Sigma$ is stably parallelisable by [KM], Theorem 3.1] and hence admits an almost contact structure $\varphi$ with stabilisation $\zeta:=S \varphi$. The complex normal $(q-1)$-type of $(\Sigma, \zeta)$ is independent of the choice of $\varphi$ and is given by

$$
\left(B_{\zeta}^{q-1}, \eta_{\zeta}^{q-1}\right)=\left(B U\langle q+1\rangle, \pi_{q+1}\right),
$$

where $\pi_{q+1}: B U\langle q+1\rangle \rightarrow B U$ is the $q^{t h}$ connective cover of $B U$ : see Example 2.5.

For homotopy spheres bounding parallelisable manifolds we have the following well-known proposition.

Proposition 5.3. Every homotopy sphere $\Sigma \in b P_{2 q+2}$ is Stein fillable.

Proof. In order to exhibit an explicit Stein filling for $\Sigma$, we use the fact that every $\Sigma \in b P_{2 q+2}$ is diffeomorphic to a 'Brieskorn sphere', [Br , Korollar 2]. That is, $\Sigma \cong \Sigma\left(a_{1}, a_{2}, \ldots, a_{q+2}\right)$ is realised as the intersection of the singular hypersurface

$$
H_{0}=\left\{\left(z_{1}, \ldots, z_{2 q+2}\right) \mid z_{1}^{a_{1}}+z_{2}^{a_{2}}+\ldots+z_{q+2}^{a_{q+2}}=0\right\} \subset \mathbb{C}^{q+2}
$$

with the unit sphere $S^{2 q+3} \subset \mathbb{C}^{q+2}$ for suitable $a_{i} \in \mathbb{N}$. A Stein filling is then given by considering the part of a regular hypersurface,

$$
H_{\epsilon}=\left\{\left(z_{1}, \ldots, z_{2 q+2}\right) \mid z_{1}^{a_{1}}+z_{2}^{a_{2}}+\ldots+z_{q+2}^{a_{q+2}}=\epsilon\right\},
$$

that intersects the unit ball $B^{2 q+4} \subset \mathbb{C}^{q+2}$ for any small $\epsilon \neq 0$ and the strictly plurisubharmonic function is given by $\|z\|^{2}$.

When we move to homotopy $(2 q+1)$-spheres mapping non-trivially to Coker $\left(J_{2 q+1}\right)$, there is no known example admitting a Stein filling. The following proposition, which is a more precise version of Theorem 1.4 from the introduction, is a consequence of Theorem 3.3 as well as results of Wall and Schultz about homotopy spheres bounding highly-connected manifolds.

Theorem 5.4. Let $\Sigma^{2 q+1}$ be a homotopy sphere which maps non-trivially into $\operatorname{Coker}\left(J_{2 q+1}\right)$.

(1) If $q \not \equiv 1,3,7 \bmod 8$ or if $q \equiv 1 \bmod 8$ and $q>9$ or if $q=7$ or 15 , then $\Sigma$ is not Stein fillable.

(2) If $q=9$ or if $q \equiv 3,7 \bmod 8$, then there is a cyclic subgroup $C_{q}^{U} \subset \operatorname{Coker}\left(J_{2 q+1}\right)$ such that $\Sigma$ is Stein fillable if and only if $\Sigma$ maps to zero in $\operatorname{Coker}\left(J_{2 q+1}\right) / C_{q}^{U}$.

(a) For $q=9$, we have $C_{9}^{U} \cong 0$ or $\mathbb{Z}_{2}$.

(b) For $q \equiv 7 \bmod 8$, we have $C_{8 k-1}^{U} \subset 4 \cdot \operatorname{Coker}\left(J_{16 k-1}\right)$.

There are many cases where the group $\operatorname{Coker}\left(J_{2 q+1}\right) / C_{q}^{U}$ is non-zero: we discuss some examples in Corollary 5.6 and Lemma 5.8 below. By the Generalized Poincaré Conjecture, Theorem 5.1 and Theorem 5.4 imply the following

Corollary 5.5. In general, the existence of a Stein fillable contact structure depends on the smooth structure of $M$ and not simply the underlying homeomorphism type of $M$.

Proof of Theorem 5.4. Let $(W, J)$ be a Stein filling of $\Sigma^{2 q+1}$. Since $W$ has handles only in dimension $(q+1)$ or less, it follows that $W$ is obtained from $\Sigma$ by attaching handles of dimension $(q+1)$ or greater. Hence $W$ is $q$-connected and so $\Sigma$ bounds a $q$-connected smooth manifold $W$ with a stable complex structure $\bar{\zeta}_{W}$. This constrains the diffeomorphism type of $\Sigma$ as recorded in the statement of the proposition, as we now explain. 
The classification of oriented $q$-connected $(2 q+2)$-manifolds with boundary a homotopy sphere is given in [Wa1]. Such manifolds are homotopy equivalent to a finite wedge of $(q+1)$-spheres and are classified by triples

$$
(H, \lambda, \alpha)=\left(H_{q+1}(W), \lambda_{W}, \alpha_{W}\right)
$$

where $\left(H_{q+1}(W), \lambda_{W}\right)$ is the usual intersection form of $W$, which is a unimodular bilinear form over the integers, and $\alpha_{W}: H_{q+1}(W) \rightarrow \pi_{q}(S O(q+1))$ is a quadratic refinement of $\lambda_{W}$ as explained in [Wa1, Lemma 2]. The stablisation of $\alpha_{W}$ is a homomorphism

$$
S \alpha_{W}: H_{q+1}(W) \rightarrow \pi_{q}(S O)
$$

which describes the stable tangent bundle of $W$ along each $(q+1)$-sphere in the homotopy type of $W$. In particular, $W$ admits a complex structure if and only if

$$
\operatorname{Im}\left(S \alpha_{W}\right) \subset \operatorname{Im}\left(\pi_{q}(U) \rightarrow \pi_{q}(S O)\right) .
$$

To study the diffeomorphism type of the homotopy sphere $\Sigma=\partial W$, Wall Wa1, Theorems $2 \& 3$ ] defined the bordism group

$$
A_{2 q+2}^{\langle q+1\rangle}:=\{[W] \mid W \text { is } q \text {-connected and } \partial W \cong \Sigma\},
$$

the rel. boundary bordism group of smooth oriented $q$-connected $(2 q+2)$-manifolds with boundary a homotopy sphere. (The notation is from [Sto] and a similar notation appears in [Wa2, $\S 17]$.) In analogy, we define the bordism group

$$
A_{2 q+2}^{U\langle q+1\rangle}:=\{[W, J] \mid W \text { is } q \text {-connected and } \partial W \cong \Sigma\},
$$

to be the rel. boundary bordism group of almost complex q-connected $(2 q+2)$-manifolds with boundary a stably complex homotopy sphere. We consider the homomorphisms

$$
A_{2 q+2}^{U\langle q+1\rangle} \stackrel{F}{\longrightarrow} A_{2 q+2}^{\langle q+1\rangle} \stackrel{\partial}{\longrightarrow} \Theta_{2 q+1} \stackrel{\eta}{\longrightarrow} \operatorname{Coker}\left(J_{2 q+1}\right)
$$

where $F$ remembers only the orientation underlying an almost complex structure, $\partial$ is defined by taking the diffeomorphism type of the bounding homotopy sphere, and $\eta$ is the homomorphism from Theorem 5.1. The above discussion shows that the group

$$
C_{q}^{U}:=\operatorname{Im}(\eta \circ \partial \circ F) \subset \operatorname{Coker}\left(J_{2 q+1}\right)
$$

is isomorphic to the group of Stein fillable homotopy spheres modulo $b P_{2 q+2}$.

Let $P_{2 q+2} \subset A_{2 q+2}^{U\langle q+1\rangle}$ denote the subgroup generated by parallelisable manifolds so that $\partial P_{2 q+2}=b P_{2 q+2}$. When $q=2 k$ is even, $\pi_{2 k}(U)=0$ and every $2 k$-connected almost complex $(4 k+2)$-manifold $W$ is parallelisable. Hence

$$
C_{2 k}=(\eta \circ \partial \circ F)\left(A_{4 k+2}^{U\langle 2 k+1\rangle}\right)=\eta\left(P_{4 k+2}\right)=0,
$$

and it remains to consider the case where $q$ is odd. Wall [Wa2, Theorem 11] computed the group $A_{2 q+2}^{\langle q+1\rangle}$ by proving that it is isomorphic to a certain Witt group of quadratic forms $(H, \lambda, \alpha)$ as above. The computation is based on [Wa2, Theorem 11] where certain Grothendieck groups which surject onto $A_{2 q+2}^{\langle q+1\rangle}$ were computed. We do not go into the details but summarise the facts relevant for our proof. By [Wa1, Theorem 4], $\eta \circ \partial=0$ for $3 \leq q \leq 7$ and hence $C_{q}^{U}=0$ in these dimensions. 
We now assume that $q=2 k+1 \geq 9$ : by [Wa2, Theorem 11] and [Wa1, Theorem 2] there is an isomorphism

$$
\Phi: A_{2 q+2}^{\langle q+1\rangle} \cong P_{2 q+2} \oplus\left(\pi_{q}(S O) \otimes \pi_{q}(S O)\right), \quad[H, \lambda, \alpha] \mapsto\left(\sigma(H, \lambda), \chi^{2}\right) .
$$

Here $\chi \in H$ is an element such that $\lambda(x, \chi)=S \alpha(x)$ for all $x \in H$, intrepreted mod 2 if $\pi_{q}(S O) \cong \mathbb{Z}_{2}$, and $\chi^{2}=\lambda(\chi, \chi)$ and we do not define $\sigma(H, \lambda) \in b P_{2 q+2}$ since $\partial\left(P_{2 q+2}\right) \subset$ $b P_{2 q+2}$ does not concern us. Let $F_{*}: \pi_{q}(U) \rightarrow \pi_{q}(S O)$ be the homomorphism induced by $U \rightarrow S O$. From (9) and (8) above, we see that there is an isomorphism

$$
\Phi^{U}: F\left(A_{2 q+2}^{U\langle q+1\rangle}\right) \cong P_{2 q+2} \oplus\left(\operatorname{Im}\left(F_{*}\right) \otimes \operatorname{Im}\left(F_{*}\right)\right) .
$$

It follows that $C_{q}^{U}$ is the zero group if $F_{*}=0$. Given our knowledge of the homomorphism $\pi_{q}(U) \rightarrow \pi_{q}(S O)$ this occurs unless $q \equiv 1,3,7 \bmod 8$. When $q \equiv 1,3,7 \bmod 8$, we see that $C_{q}^{U}$ is the cyclic group generated by the element

$$
\left(\eta \circ \partial \circ\left(\Phi^{U}\right)^{-1}\right)\left(0, F_{*}(1) \otimes F_{*}(1)\right) \in \operatorname{Coker}\left(J_{2 q+1}\right),
$$

where $1 \in \pi_{q}(U)$ is a generator. For $q \equiv 1,3 \bmod 8, F_{*}$ is onto. However, if $q=8 k+1>9$ Schultz [Sc, Corollary 3.2] states that $\eta \circ \partial=0$, proving that $C_{8 k+1}^{U}=0$ if $8 k+1>9$. Finally, for $q \equiv 7 \bmod 8, F_{*}\left(\pi_{q}(U)\right) \subset \pi_{q}(S O)$ is a subgroup of index two and so the bilinearity of the tensor product ensures that $C_{q}^{U}=4 \cdot \operatorname{Im}(\eta \circ \partial)$. When $q=15$, we have $\operatorname{Coker}\left(J_{31}\right) \cong \mathbb{Z}_{2}^{2}$, [R, Table A3.3], and hence $C_{15}^{U}=0$.

We now give some examples of exotic spheres which are not Stein fillable. Since every homotopy sphere has a unique spin structure, there is a homomorphism $\omega^{\text {Spin }}: \Theta_{n} \rightarrow \Omega_{n}^{\text {Spin }}$, given by mapping a homotopy sphere to it spin bordism class. Recall now the $\alpha$-invariant

$$
\alpha: \Omega_{*}^{\text {Spin }} \rightarrow \mathrm{KO}_{*}
$$

which is a ring homomorphism from spin bordism to real $K$-theory defined by taking the $K O$-valued index of the Dirac operator on a spin manifold, [Hi, §4.2]. Composing $\alpha$ with $\omega^{\text {Spin }}$ we obtain the $\alpha$-invariant for homotopy spheres

$$
\alpha: \Theta_{n} \rightarrow \Omega_{n}^{S p i n} \rightarrow \mathrm{KO}_{n} .
$$

It is known that in all dimensions $8 k+1, k \geq 1$, there are exotic spheres with non-trivial $\alpha$-invariant in $K O_{8 k+1} \cong \mathbb{Z}_{2}$. The existence of such spheres follows from theorems of Milnor and Adams as is explained in [Hi, p. 44]. If $\alpha(\Sigma)=1$ then $\Sigma$ does not bound a spin manifold. On the other hand, a Stein filling of $\Sigma$ is $4 k$-connected and in particular admits a unique spin structure. Hence we obtain an alternative proof of the following special case of Theorem 5.4 .

Lemma 5.6. If $\Sigma \in \Theta_{8 k+1}$ has $\alpha(\Sigma)=1 \in K O_{8 k+1}$ then $\Sigma$ is not Stein fillable.

Next we show that taking connected sums with $\alpha$-invariant-1 homotopy spheres can often destroy the Stein fillability of more general manifolds. Since $\pi_{8 k+1}(S O / U)=0$, it follows that every homotopy $(8 k+1)$-sphere has a unique stable complex structure $\zeta_{\Sigma}$. Given a stably complex manifold $(M, \zeta)$, we shall write $\left(M \sharp \Sigma, \zeta \sharp \zeta_{\Sigma}\right)$ for the stably complex manifold obtained by taking the connected sum of the stably complex manifolds $(M, \zeta)$ and $\left(\Sigma, \zeta_{\Sigma}\right)$.

Proposition 5.7. Let $(M, \varphi)$ be a Stein fillable almost contact manifold of dimension $8 k+1$ with $\zeta:=S \varphi$ and $c_{1}(\zeta)=0$. If $\Sigma$ is a homotopy $(8 k+1)$-sphere with $\alpha(\Sigma)=1 \in K O_{8 k+1}$, then the stably complex manifold $\left(M \sharp \Sigma, \zeta \sharp \zeta_{\Sigma}\right)$ is not Stein fillable. 
Proof. Since $c_{1}(\zeta)=0$, there is a lift of the normal complex structure $\zeta: M \rightarrow B U$ to $B S U$. It follows that there is a map of stable complex bundles $F:\left(B_{\zeta}^{4 k-1}, \eta_{\zeta}^{4 k-1}\right) \rightarrow\left(B S U, \pi_{S U}\right)$. The bundle map $F$ induces a homomorphism of bordism groups

$$
F_{*}: \Omega_{8 k+1}\left(B_{\zeta}^{4 k-1} ; \eta_{\zeta}^{4 k-1}\right) \rightarrow \Omega_{8 k+1}^{S U} .
$$

Since $(M, \varphi)$ is Stein fillable by Theorem 3.7, every $(4 k-1)$-smoothing $\bar{\zeta}: M \rightarrow B_{\zeta}^{4 k-1}$ is nullbordant. Moreover $\pi_{8 k+1}(S O / U)=0$ and thus $\Sigma$ admits a unique $\left(B_{\zeta}^{4 k-1}, \eta_{\zeta}^{4 k-1}\right)$-structure $\bar{\zeta}_{\Sigma}$. The connected sum $\left(M \sharp \Sigma, \bar{\zeta} \sharp \bar{\zeta}_{\Sigma}\right)$ is a $\left(\zeta \sharp \zeta_{\Sigma}\right)$-compatible normal $(4 k-1)$-smoothing in $\left(B_{\zeta}^{4 k-1}, \eta_{\zeta}^{4 k-1}\right)$. Now we have

$$
F_{*}\left(\left[M \sharp \Sigma, \bar{\zeta} \sharp \bar{\zeta}_{\Sigma}\right]\right)=\left[\Sigma, \zeta_{\Sigma}\right] \neq 0 \in \Omega_{8 k+1}^{S U},
$$

where the last inequality holds since the homomorphism $S U \rightarrow$ Spin induces a homomorphism $\Omega_{*}^{S U} \rightarrow \Omega_{*}^{S p i n}$. Since $\alpha(\Sigma)=1$, it follows that $\left[\Sigma, \zeta_{\Sigma}\right] \neq 0 \in \Omega_{8 k+1}^{S U}$. The above argument therefore shows that $\left[M \sharp \Sigma, \bar{\zeta}_{\sharp} \bar{\zeta}_{\Sigma}\right] \neq 0 \in \Omega_{8 k+1}\left(B_{\zeta}^{4 k-1} ; \eta_{\zeta}^{4 k-1}\right)$, and so by Theorem 3.7, $\left(M \sharp \Sigma, \zeta \sharp \zeta_{\Sigma}\right)$ is not Stein-fillable.

We next construct a certain exotic 9-sphere $\Sigma$ which lies inthe kernel of the $\alpha$ invariant $\alpha: \Theta_{9} \rightarrow \mathrm{KO}_{9}$, but which does not bound a parallelisable manifold. By Theorem 5.4, this homotopy sphere is not Stein fillable, but from a topological point of view, one can argue that it is one of the "least exotic" homotopy spheres which is not Stein fillable. To the best of our knowledge, it is not known whether $\Sigma$ admits a symplectically fillable contact structure.

By Theorem 5.1 above and results of Toda [To, p. 189], there is a short exact sequence

$$
0 \rightarrow b P_{10} \rightarrow \operatorname{Ker}(\alpha) \rightarrow \mathbb{Z}_{2} \rightarrow 0 \text {. }
$$

We shall given a explicit description of a homotopy sphere $\Sigma$ where $[\Sigma]$ generates $\operatorname{Ker}(\alpha) / b P_{10}$. We first recall the well-known plumbing pairing

$$
\sigma_{p, q}: \pi_{p}(S O(q)) \times \pi_{q}(S O(p)) \longmapsto \Theta_{p+q+1}, \quad(\beta, \gamma) \longmapsto \partial W(S(\beta), S(\gamma)),
$$

where $S: \pi_{p}(S O(q)) \rightarrow \pi_{p}(S O(q+1))$ is the the stabilisation homomorphism and

$$
W(S(\beta), S(\gamma)):=\left(D^{q+1} \tilde{\times}_{S(\beta)} S^{p+1}\right) \cup_{D^{q+1} \times D^{p+1}}\left(D^{p+1} \tilde{\times}_{S(\gamma)} S^{q+1}\right)
$$

is the compact smooth $(p+q+2)$-manifold obtained by plumbing the disc bundles of $S(\beta)$ and $S(\gamma)$ together: see for example [Sc, Remark p. 741]. We let $\beta_{5} \in \pi_{3}(S O(5)) \cong \mathbb{Z}$ and $\gamma_{3} \in \pi_{5}(S O(3)) \cong \mathbb{Z}_{2}$ be generators and define the homotopy 9 -sphere

$$
\Sigma_{\beta_{5}, \gamma_{3}}^{9}:=\sigma_{3,5}\left(\beta_{5}, \gamma_{3}\right) \text {. }
$$

Notice that there is a homotopy equivalence $W\left(S\left(\beta_{5}\right), S\left(\gamma_{3}\right)\right) \simeq S^{4} \vee S^{6}$, so that the manifold $W\left(S\left(\beta_{5}\right), S\left(\gamma_{3}\right)\right)$ cannot admit a Stein structure, but from the point of view of the dimensions of the handles, $W\left(S\left(\beta_{5}\right), S\left(\gamma_{3}\right)\right)$ is as close as possible to admitting a Stein structure.

Lemma 5.8. The homotopy 9 -sphere $\Sigma_{\beta_{5}, \gamma_{3}}$ maps to a generator of $\operatorname{Ker}(\alpha) / b P_{10} \cong \mathbb{Z}_{2}$.

Proof. The proof starts with the exotic 8-sphere $\Sigma^{8} \in \Theta_{8} \cong \mathbb{Z}_{2}$. By [Sto, Satz 12.1] and [Hu, Proposition 12.20], there is a diffeomorphism $\Sigma^{8} \cong \partial W\left(\beta_{5}, \delta_{4}\right)$ where $\beta_{5} \in \pi_{3}(S O(5))$ is as above and $\delta_{4} \in \pi_{4}(S O(4))$ is given by the composition $\tau_{S^{4}} \circ \eta_{3}: S^{4} \rightarrow S^{3} \rightarrow S O(4)$, where $\tau_{S^{4}}$ is the characteristic map of the tangent bundle of the 4 -sphere and $\eta_{3}$ is essential. We 
claim that $\delta_{4}=S\left(\delta_{3}\right)$ where $\delta_{3} \in \pi_{4}(S O(3)) \cong \mathbb{Z}_{2}$ is a generator. To see this, we use the commutative diagram of exact sequences

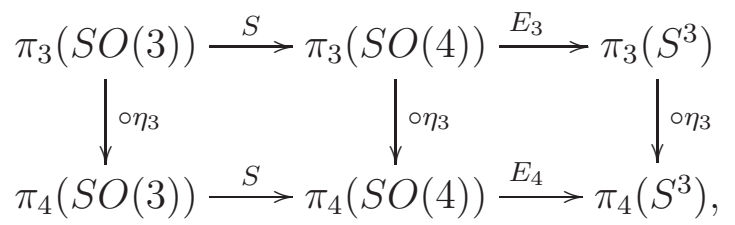

where the horizontal sequences are part of the homotopy long exact sequence of the fibration $S O(3) \rightarrow S O(4) \rightarrow S^{3}$, the vertical maps are given by pre-composition with $\eta_{3}$, and the map $E_{3}$ takes the Euler class of the corresponding bundle. Since $E_{3}\left(\tau_{S^{4}}\right)= \pm 2 \in \pi_{3}\left(S^{3}\right) \cong \mathbb{Z}$, it follows that $E_{3}\left(\tau_{S^{4}}\right) \circ \eta_{3}=0$ and so $E_{4}\left(\tau_{S^{4}} \circ \eta_{3}\right)=0$. Hence $\tau_{S^{4}} \circ \eta_{3} \in \operatorname{Im}(S)$. Since $\Sigma^{8}$ is non-standard, $\tau_{S^{4}} \circ \eta_{3}$ is non-zero and this proves the claim. It follows that $\Sigma^{8} \cong \sigma_{3,4}\left(\beta_{4}, \delta_{3}\right)$, where $\beta_{4} \in \pi_{3}(S O(4))$ stabilises to $\beta_{5}$.

To relate $\Sigma^{8}$ to $\Sigma_{\beta_{5}, \gamma_{3}}$ we shall use the Milnor-Munkres-Novikov pairing [La2, p. 583],

$$
\tau_{p, q}: \pi_{p}\left(S O_{q}\right) \times \Theta_{q} \longmapsto \Theta_{p+q}, \quad(\alpha, \Sigma) \longmapsto \partial W(\alpha, \Sigma),
$$

where $W(\alpha, \Sigma)$ is the plumbing manifold

$$
\left(D^{q} \tilde{\times}_{\alpha} S^{p+1}\right) \cup_{D^{p+1} \times D^{q}}\left(D^{p+1} \times \Sigma^{q}\right)
$$

obtained by plumbing the disc bundle of $\alpha$ with the trivial $(p+1)$-disc bundle over the homotopy sphere $\Sigma$. By [Sc, Theorem 2.5], if $\mu_{n} \in \pi_{1}(S O(n)) \cong \mathbb{Z}_{2}$ is a generator for $n \geq 3$, then

$$
\left.\tau_{1,8}\left(\mu_{8}, \sigma_{3,4}\left(\beta_{4}, \delta_{3}\right)\right)\right)=\sigma_{3,5}\left(S \beta_{4}, \delta_{3} \circ \eta_{4}\right)
$$

so long as the Samelson product $S\left(\beta_{4}\right) * S\left(\mu_{4}\right) \in \pi_{4}(S O(5))$ is trivial: we assume this for now and complete the proof. Since $\gamma_{3}=\delta_{3} \circ \eta_{4}$, it follows that $\Sigma_{\beta_{5}, \gamma_{3}} \cong \tau_{1,8}\left(\mu_{8}, \Sigma^{8}\right)$. But it is clear from the definition of the pairing $\tau_{p, q}$ that $\eta\left(\tau_{1,8}\left(\mu_{8}, \Sigma_{8}\right)\right)=\left[\eta\left(\Sigma^{8}\right) \circ \eta_{8}\right] \in \operatorname{Coker}\left(J_{9}\right)$. But by [To, p. 189], $\left[\eta\left(\Sigma^{8}\right) \circ \eta_{8}\right] \neq 0 \in \operatorname{Coker}\left(J_{9}\right)$ and so $\Sigma_{\beta_{5}, \gamma_{3}}$ does not belong to $b P_{10}$. On the other hand, $\Sigma_{\beta_{5}, \gamma_{3}}$ bounds a spin manifold by construction and so $\Sigma_{\beta_{5}, \beta_{3}} \in \operatorname{Ker}(\alpha)$.

To complete the proof, we must show that the Samelson product $\beta_{5} * \mu_{5}$ vanishes. It suffices to show that $\beta_{4} * \mu_{4}$ vanishes. Recall that the Samelson product $\beta_{4} * \mu_{4}: S^{4} \rightarrow S O(4)$ is defined to be the homotopy class of the map induced on $S^{4}$ by the following map

$$
S^{3} \times S^{1} \rightarrow S O(4), \quad(x, \lambda) \mapsto \beta_{4}(x) \mu_{4}(y) \beta_{4}^{-1}(x) \mu_{4}^{-1}(y) .
$$

Now, we represent $\beta_{4}$ and $\mu_{4}$ by the following maps:

$$
\beta_{4}(x)(y)=x \cdot y \quad \text { and } \quad \mu_{4}(\lambda)(y)=y \cdot \lambda,
$$

where $y \in \mathbb{H}$ is a quaternion, $x \in S^{3}$ a unit quaternion and $\lambda \in S^{1} \subset S^{3}$ a unit complex number. Evidently $\beta_{4}(x), \mu_{4}(\lambda) \in S O(4)$ commute for all values of $(x, \lambda)$ and hence the Samelson product $\beta_{4} * \mu_{4}$ vanishes.

By Lemma 5.3 homotopy spheres in $b P_{2 q+2} \subset \Theta_{2 q+1}$ (i.e. the ones mapping trivially to $\left.\operatorname{Coker}\left(J_{2 q+1}\right)\right)$ are all Stein fillable, while Theorem 5.4 shows that many homotopy spheres with non-trivial image in $\operatorname{Coker}\left(J_{2 q+1}\right)$ do not admit Stein fillings. This observation naturally leads us to the following

Conjecture 5.9. A homotopy sphere $\Sigma^{2 q+1}$ is Stein fillable if and only $\Sigma^{2 q+1} \in b P_{2 q+2}$. That is, in the notation of Theorem 5.4. $C_{q}^{U}=0$ for all $q$. 
Notice that while Theorem 5.4 shows that many exotic spheres are not Stein fillable, those same homotopy spheres might admit symplectically fillable contact structures.

Problem 5.10 (Symplectic fillability of homotopy spheres). Do all homotopy spheres admit symplectically fillable contact structures? If not, then determine all those that do.

The positive resolution of this problem would imply that symplectic fillability is invariant under the action of the group of exotic spheres under connect sum. Notice that although our Filling Theorem 3.7 is not useful in searching for symplectic fillings which are not also Stein fillings, Corollary 3.10 may be helpful in finding symplectically fillable contact structures on homotopy spheres which do not admit Stein fillings.

\section{Further Properties of Stein Fillable MANifolds}

In this section we discuss several topological properties of Stein fillable manifolds.

6.1. (Co)homological obstructions to Stein fillability. In this subsection we discuss topological obstructions to Stein fillability, which are not present in dimension 3, and some of their consequences. (See also [PP] and [EKP] for similar obstructions.) As usual, let $(M, \varphi)$ be an almost contact manifold with associated stable complex structure $\zeta$, let $\left(B_{\zeta}^{q-1}, \eta_{\zeta}^{q-1}\right)$ be the complex normal $(q-1)$-type of $(M, \zeta)$ and let $\bar{\zeta}: M \rightarrow B_{\zeta}^{q-1}$ be a $\zeta$-compatible normal $(q-1)$-smoothing. We begin by observing that there is a commutative diagram,

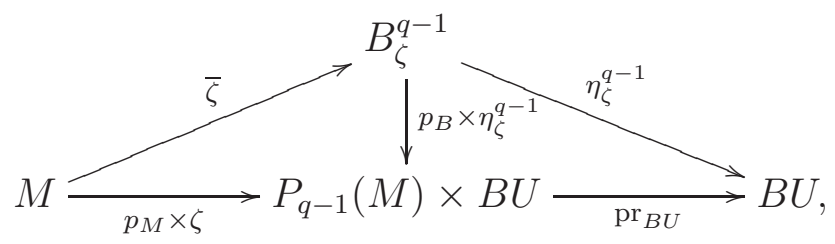

where $P_{q-1}(B) \simeq P_{q-1}(M)$ is the $(q-1)^{s t}$ Postnikov stage of $M$ and $B$, and the maps $p_{M}: M \rightarrow P_{q-1}(M)$ and $p_{B}: B \rightarrow P_{q-1}(M)$ are $q$-equivalences. We see that the induced homomorphism

$$
\left(p_{B} \times \eta_{\zeta}^{q-1}\right)_{*}: \Omega_{2 q+1}\left(B_{\zeta}^{q-1} ; \eta_{\zeta}^{q-1}\right) \rightarrow \Omega_{2 q+1}^{U}\left(P_{q-1}(M)\right)
$$

is such that $\left(p_{B} \times \eta_{\zeta}^{q-1}\right)_{*}([M, \bar{\zeta}])=\left[(M, \zeta), p_{M}\right]$. Applying Theorem 3.7 we obtain

Lemma 6.1. If $\left[(M, \zeta), p_{M}\right] \neq 0 \in \Omega_{2 q+1}^{U}\left(P_{q-1}(M)\right)$, then $(M, \zeta)$ does not admit a Stein fillable contact structure.

The following proposition combines Lemma 6.1 with other elementary observations to give obstructions to Stein fillability. Let $\pi=\pi_{1}(M)$ denote the fundamental group of $M$.

Proposition 6.2. Suppose that $(M, \varphi)$ is an almost contact manifold of dimension $2 q+1 \geq 5$ that admits a Stein fillable contact structure and let $u: M \rightarrow K(\pi, 1)$ be the classifying map of the universal cover of $M$. Then the following hold:

(1) The homomorphism $u_{*}: H_{i}(M ; \mathbb{Z}) \rightarrow H_{i}(K(\pi, 1))$ vanishes for $q+2 \leq i \leq 2 q+1$. In particular $u_{*}([M])=0 \in H_{2 q+1}(K(\pi, 1))$, where $[M]$ denotes the fundamental class of $M$.

(2) $M$ is not aspherical. 
(3) For any $\beta \in H^{j}\left(P_{q-1}(M)\right)$ and for any $k$-tuple $\left\{i_{1}, \ldots, i_{k}\right\}$ of positive integers with $2 q+1-j=2\left(\sum_{n=1}^{k} i_{n}\right)$, all products of the form $p_{M}^{*}(\beta) \cup c_{i_{1}}(\zeta) \cup \cdots \cup c_{i_{k}}(\zeta) \in$ $H^{2 q+1}(M)$ vanish.

(4) For any $k$-tuple $\left\{i_{1}, \ldots, i_{k}\right\}$ of positive integers with $\sum_{n=1}^{k} i_{n}=q$, all products of Chern classes $c_{i_{1}}(\zeta) \cup \cdots \cup c_{i_{k}}(\zeta) \in H^{2 q}(M)$ vanish.

Proof. (1) Let $\left(W, \bar{\zeta}_{W}\right)$ be a $B$-nullbordism of $(M, \bar{\zeta})$. After surgery we may assume that $W$ has no handles in dimension greater than $q+1$ and hence $H_{i}(W ; \mathbb{Z})=0$ for $i>q+1$. Now $\bar{\nu}: M \rightarrow B$ factors over $W$ and $u: M \rightarrow K(\pi, 1)$ can be factored as $u_{B} \circ \bar{\nu}: M \rightarrow B \rightarrow$ $K(\pi, 1)$, where $u_{B}: B \rightarrow K(\pi, 1)$ classifies the universal covering of $B$.

(2) If $M$ is aspherical then $M \simeq K(\pi, 1)$ and so $u_{*}([M])$ is a generator of the group $H_{2 q+1}(K(\pi, 1)) \cong \mathbb{Z}$. Now apply part $(1)$.

(3) The integer

$$
\left\langle p_{M}^{*}(\beta) \cup c_{i_{1}}(\zeta) \cup \cdots \cup c_{i_{k}}(\zeta),[M]\right\rangle
$$

is an invariant of unitary bordism of $P_{q-1}(M)$. By Lemma 6.1 this integer vanishes for the unitary $P_{q-1}(M)$-manifold $\left((M, \zeta), p_{M}\right)$. Since $H^{2 q+1}(M) \cong \mathbb{Z}$, this finishes the proof.

(4) We apply part (3) with $\beta \in H^{1}\left(P_{q-1}(M)\right)=H^{1}(M)$ and then use a version of part (1) with $\bmod \mathbb{Z} / p$ coefficients to conclude that $c_{i_{1}}(\zeta) \cup \cdots \cup c_{i_{k}}(\zeta)$ vanishes in $H^{2 q}(M ; \mathbb{Q})$ and also in $H^{2 q}(M ; \mathbb{Z} / p)$ for all primes $p$. It follows that $c_{i_{1}}(\zeta) \cup \cdots \cup c_{i_{k}}(\zeta)=0 \in H^{2 q}(M)$.

Proposition 6.2 allows us to prove the following

Corollary 6.3. In general, the Stein fillability of an almost contact manifold $(M, \varphi)$ depends on the choice $\varphi$ and not just the underlying diffeomorphism type of $M$.

Proof. The manifold $M=S^{1} \times S^{6}$ clearly admits a Stein fillable almost contact structure $\varphi_{0}$ since $M=\partial\left(S^{1} \times D^{7}\right)$. On the other hand, $S^{6}$ admits an almost complex structure $J$ with $c_{3}(J)=2 \in H^{6}\left(S^{6}\right)$. For the induced almost contact structure $\varphi_{1}$ on $M$, Proposition 6.2 (44) implies that $\left(M, \varphi_{1}\right)$ is not Stein fillable.

As a consequence of Proposition 6.2, we obtain obstructions to the Stein fillability of certain Boothby-Wang contact structures.

Example 6.4 (Boothby-Wang contact structures). A Boothby-Wang contact structure on a (nontrivial) principal $S^{1}$-bundle

$$
S^{1} \longrightarrow E \stackrel{\pi}{\longrightarrow} B
$$

over a symplectic base $(B, \omega)$ of dimension $2 q$ with $c_{1}(E)=\left[\frac{\omega}{2 \pi}\right]$ is given as the kernel of an $S^{1}$-invariant 1-form $\alpha$ which is non-vanishing on the fibers and satisfies $d \alpha=\pi^{*}(\omega)$ for some integral symplectic form $\omega$.

Note that the associated disc bundle of the principal $S^{1}$-bundle $E$ is a strong symplectic filling (see, e.g., GS], Lemma 3), which is not Stein since it is homotopy equivalent to the $2 q$-dimensional base $B$. (However, if the base is $\mathbb{C} P^{2}$ and the Euler class of the bundle is a generator of $H^{2}\left(\mathbb{C} P^{2} ; \mathbb{Z}\right)$, then the total space is the 5 -sphere which is of course Stein fillable.) On the other hand we do have the following example:

Example 6.5 (Lens spaces). Let $L_{k}^{5}$ be the standard 5-dimensional lens space with cyclic fundamental group of order $k$. That is, $L_{k}^{5}$ is the quotient of

$$
S^{5}=\left\{\left.\left(z_{1}, z_{2}, z_{3}\right) \in \mathbb{C}^{3}|| z_{1}\right|^{2}+\left|z_{2}\right|^{2}+\left|z_{3}\right|^{2}=1\right\},
$$


with the action of a generator of $\mathbb{Z}_{k}$ defined by

$$
\left(z_{1}, z_{2}, z_{3}\right) \mapsto\left(\mu z_{1}, \mu z_{2}, \mu z_{3}\right)
$$

for $\mu \in \mathbb{C}$ a $k^{t h}$ root of unity. The resulting manifold inherits an $S^{1}$-bundle projection $\pi: L_{k}^{5} \rightarrow \mathbb{C} P^{2}$. Since the classifying map of the universal cover $u$ induces a non-trivial map

$$
u_{*}: H_{i}\left(L_{k}^{5}\right) \rightarrow H_{i}\left(K\left(\mathbb{Z}_{k}, 1\right)\right),
$$

we conclude that although the lens spaces $L_{k}^{5}$ are symplectically fillable (by the BoothbyWang construction), by Lemma 6.2 (11) they are not Stein fillable for all $k \geq 2$. (Obstructions for Stein fillability of these manifolds were already noticed in [EKP], cf. also [PP].)

In conclusion, we see both examples of Boothby-Wang contact structures which are Stein fillable, and others which are not. This observation leads to the following question:

Problem 6.6 (Fillability of Boothby-Wang manifolds). Determine which Boothby-Wang manifolds are Stein/exactly fillable.

By recent work of Massot, Niederkrüger and Wendl [MNW], Proposition 6.2 also gives examples of exactly fillable contact structures that are not Stein fillable in all dimensions. Such examples were discussed in Bow for 3-dimensional manifolds, although in this case the nonfillability only applied to certain contact structures rather than to the manifolds themselves. We are now in the position to provide the proof of Theorem 1.5 from the Introduction:

Proof of Theorem 1.5. By [MNW, Theorem C], there are exact symplectic fillings of the form $M \times[0,1]$ such that both ends are convex in all dimensions $2 q+2$. The manifolds $M$ are quotients of contractible Lie groups and are consequently aspherical. After attaching a Weinstein 1-handle to $M \times[0,1]$, we obtain an exact filling of $N=-M \# M$. Assuming that $q>1, \pi_{1}(N)$ is the free product two copies of $\pi_{1}(M)$ and so there is a homotopy equivalence $K\left(\pi_{1}(N), 1\right) \simeq K\left(\pi_{1}(M), 1\right) \vee K\left(\pi_{1}(M), 1\right)$. Since $M$ is aspherical, we see that the classifying map of the universal cover of $N$ maps non-trivially on $H_{2 q+1}(N)$. Hence by Proposition 6.2 (1), $N$ is not Stein fillable if $q>1$.

6.2. Stein fillability and orientations. A cooriented contact structure $\xi=\operatorname{ker}(\alpha)$ determines an orientation of the underlying $(2 q+1)$-manifold $M$, since the form $\alpha \wedge(d \alpha)^{q}$ is nowhere vanishing. When we speak of an oriented manifold admitting a contact structure, we mean that the orientation determined by the contact structure is the given one. Moreover, if the dimension of $M$ is of the form $4 k+1$, and hence the dimension of the Stein filling of $M$ is of the form $4 k+2$, then taking the conjugate complex structure on $W$ reverses orientations. The resulting Stein fillable contact structure then gives the opposite coorientation of $\xi$, i.e. replaces $\alpha$ by $-\alpha$, which in turn swaps the orientation determined by the contact structure. So in these dimensions it is clear that $M$ is Stein fillable if and only if $-M$ is.

However, if the dimension of $M$ is $4 k+3$, then it is not immediately clear that $M$ is Stein fillable if and only if $-M$ is Stein fillable: indeed the statement is false in dimension 3, with many examples given by Seifert fibred spaces, the most famous of which is the Poincaré homology sphere [Li]. On the other hand, Eliashberg's $h$-principle implies the following

Proposition 6.7. Let $(M, \varphi)$ be an almost contact $(2 q+1)$-dimensional manifold with $q \geq 2$ and associated stable complex strcovucture $\zeta$. Then $(M, \zeta)$ is Stein fillable if and only if $(-M,-\zeta)$ is. 
Proof. The fact that any Stein filling $W$ of $(M, \zeta)$ is a manifold with boundary means that $T W$ admits a nonvanishing section and thus as complex bundles

$$
(T W, J) \cong\left(E,\left.J\right|_{E}\right) \oplus \underline{\mathbb{C}} .
$$

We then define an almost complex structure $\bar{J}$ by taking $\left.J\right|_{E}$ on $E$ and the conjugate complex structure on $\underline{\mathbb{C}}$. The almost complex structure $\bar{J}$ then induces the orientation $-W$, and applying Eliashberg's $h$-principle gives a Stein fillable contact structure on $-M$ with associated stable complex structure $-\zeta$.

\section{Subcritical Stein fillings and Stein fillings of products}

We fix a closed almost contact $(2 q+1)$-manifold $(M, \varphi)$ and as usual we let $\zeta=S \varphi$ denote the stable complex structure induced by the almost contact structure $\varphi$. A subcritical Stein filling of $(M, \varphi)$ is a Stein filling $(W, J)$ of $(M, \varphi)$ where $W$ admits a handle decomposition with handles of dimension $q$ and less. Subcritical Stein fillings have special properties; see CE].

Another filling question is the following: suppose that $\left(F, J_{F}\right)$ is an almost complex structure on a closed, oriented surface $F$. Then we can ask if the product almost contact manifold $\left(M \times F, \varphi \times J_{F}\right)$ admits a Stein filling. It is easy to see that if $(M, \varphi)$ has a subcritical Stein filling, then $\left(M \times F, \varphi \times J_{F}\right)$ is Stein fillable: if $\left(W, J_{W}\right)$ is the subcritical filling of $(M, \varphi)$ then $\left(W \times F, J_{W} \times J_{F}\right)$ is an almost complex manifold with boundary $\left(M \times F, \varphi \times J_{F}\right)$ which admits a handle decomposition with handles of dimension $q+2$ and less (and the dimension of $W \times F$ is $2 q+4)$, therefore Eliashberg's $h$-principle implies the result.

We shall further relate the two questions about Stein fillings to the bordism theory of $\left(B_{\zeta}^{q}, \eta_{\zeta}^{q}\right)$, the complex normal $q$-type of $(M, \zeta)$. We pose five related questions:

(A) When does $(M, \varphi)$ admit a subcritical Stein filling?

(B) When does $\left(M \times F, \varphi \times J_{F}\right)$ admit a Stein filling?

(C) When does $[M, \bar{\zeta}]=0 \in \Omega_{2 q+1}\left(B_{\zeta}^{q} ; \eta_{\zeta}^{q}\right)$ hold?

(D) When does $\bar{\zeta}_{*}([M])=0 \in H_{2 q+1}\left(B_{\zeta}^{q}\right)$ hold?

(E) When does $T H_{q}(M)$, the torsion subgroup of $H_{q}(M)$, vanish?

We next graphically summarise the relationship between positive answers to the questions above, writing $g(F)>0$ for the case where $F$ has positive genus; see Theorem 7.1 below.

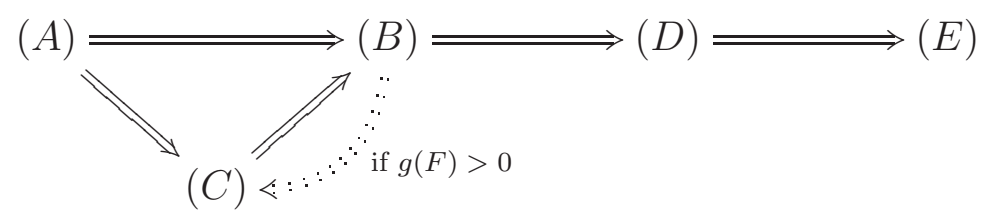

Theorem 7.1 (Subcritical Filling Theorem). Let $\left(B_{\zeta}^{q}, \eta_{\zeta}^{q}\right)$ be the complex normal q-type of $(M, \zeta)$ and let $\bar{\zeta}: M \rightarrow B_{\zeta}^{q}$ be any $\zeta$-compatible normal $q$-smoothing. If $q \geq 2$, then the following hold.

(1) If $(M, \varphi)$ admits a subcritical filling then $[M, \bar{\zeta}]=0 \in \Omega_{2 q+1}\left(B_{\zeta}^{q} ; \eta_{\zeta}^{q}\right)$.

(2) If $[M, \bar{\zeta}]=0 \in \Omega_{2 q+1}\left(B_{\zeta}^{q} ; \eta_{\zeta}^{q}\right)$ then $\left(M \times F, \varphi \times J_{F}\right)$ admits a Stein filling.

(3) If $\left(M \times F, \varphi \times J_{F}\right)$ admits a Stein filling and $g(F)>0$, then the bordism class $[M, \bar{\zeta}]$ satisfies $[M, \bar{\zeta}]=0 \in \Omega_{2 q+1}\left(B_{\zeta}^{q} ; \eta_{\zeta}^{q}\right)$. In particular, $(M, \zeta)$ is Stein fillable.

(4) If $\left(M \times F, \varphi \times J_{F}\right)$ admits a Stein filling then $\bar{\zeta}_{*}([M])=0 \in H_{2 q+1}\left(B_{\zeta}^{q}\right)$. 
(5) If $\bar{\zeta}_{*}([M])=0 \in H_{2 q+1}\left(B_{\zeta}^{q}\right)$ then $T H_{q}(M)=0$.

Proof. (1) The proof is similar to the proof of part (1) of Lemma 2.19, Let $\left(W, \zeta_{W}\right)$ denote the subcritical filling with its induced stable complex structure; it is built from $(M, \zeta)$ by adding handles with stable complex structure of dimension $q+2$ and higher. Therefore the complex normal $q$-type of $M$ can be identified with that of $W$ and the claim follows.

(2) Let $\zeta_{F}$ be the stable normal complex structure defined by $J_{F}$, let $P_{q}(F)$ be the $q^{\text {th }}$ Postnikov stage of $F$ and let $p_{F}: F \rightarrow P_{q}(F)$ be a $(q+1)$-equivalence (if $g(F)>0$, then $P_{q}(F)=K\left(\pi_{1}(F), 1\right)$ ), and let $L_{\zeta_{F}}$ be the unique complex line bundle over $P_{q}(F)$ such that $c_{1}\left(\zeta_{F}\right)=p_{F}^{*}\left(c_{1}\left(L_{\zeta_{F}}\right)\right)$. The complex normal $q$-type of $\left(M \times F, \zeta \times \zeta_{F}\right)$ is given by

$$
\left(B_{\zeta \times \zeta_{F}}^{q}, \eta_{\zeta \times \zeta_{F}}^{q}\right)=\left(B_{\zeta}^{q} \times P_{q}(F), \eta_{\zeta}^{q} \oplus L_{\zeta_{F}}\right)
$$

where, as in Section 2.5. $\eta_{\zeta}^{q} \oplus L_{\zeta_{F}}$ denotes the exterior Whitney sum of stable complex bundles. By assumption there is a $\left(B_{\zeta}^{q}, \eta_{\zeta}^{q}\right)$-null bordism $\left(W, \bar{\zeta}_{W}\right)$ of $(M, \bar{\zeta})$. We observe that

$$
\bar{\zeta}_{W} \times p_{F}: W \times F \rightarrow B_{\zeta}^{q} \times P_{q}(F)
$$

is a $\left(B_{\zeta \times \zeta_{F}}^{q}, \eta_{\zeta}^{q} \times L_{\zeta_{F}}\right)$-nullbordism of $\left(M \times F, \zeta \times \zeta_{F}\right)$. By Theorem 3.7, $\left(M \times F, \varphi \times J_{F}\right)$ is Stein fillable.

(3) If $g(F)>0$, then $F$ is a $K(\pi, 1)$ manifold and $P_{q}(F)=F$. It follows that the complex normal $q$-type of $\left(M \times F, \zeta \times \zeta_{F}\right)$ is given by

$$
\left(B_{\zeta \times \zeta_{F}}^{q}, \eta_{\zeta \times J_{F}}^{q}\right)=\left(B_{\zeta}^{q} \times F, \eta_{\zeta}^{q} \oplus L_{\zeta_{F}}\right),
$$

where $L_{\zeta_{F}}$ is defined as in the proof of (2). There is a canonical isomorphism of bordism groups

$$
\theta: \Omega_{*}\left(B_{\zeta}^{q} \times F ; \eta_{\zeta}^{q} \oplus \zeta_{F}\right) \cong \Omega_{*}^{\left(B_{\zeta}^{q} ; \eta_{\zeta}^{q}\right)}\left(F ; L_{\zeta_{F}}\right)
$$

with range the $\zeta_{F}$-twisted $\left(B_{\zeta}^{q}, \eta_{\zeta}^{q}\right)$-bordism group of $F$. Taking the transverse inverse image of a point $x \in F$ defines a homomorphism

$$
\pitchfork: \Omega_{*}^{\left(B_{\zeta}^{q} ; \eta_{\zeta}^{q}\right)}\left(F ; L_{\zeta_{F}}\right) \rightarrow \Omega_{*-2}\left(B_{\zeta}^{q} ; \eta_{\zeta}^{q}\right)
$$

On the other hand, taking the product with $\left(F, \zeta_{F}\right)$ defines a homomorphism

$$
\Pi: \Omega_{*}\left(B_{\zeta}^{q} ; \eta_{\zeta}^{q}\right) \rightarrow \Omega_{*+2}\left(B_{\zeta}^{q} \times F ; \eta_{\zeta}^{q} \oplus \zeta_{F}\right), \quad\left[X, \zeta_{X}\right] \mapsto\left[X \times F, \zeta_{X} \times \zeta_{F}\right] .
$$

From the definitions of the above homomorphisms, we see that there is a commutative diagram

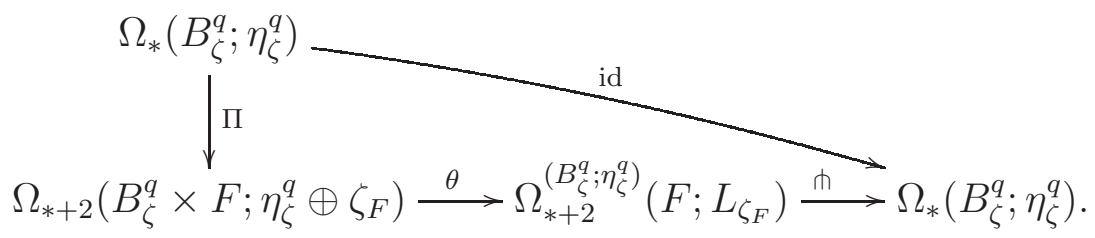

If $\left(M \times F, \varphi \times J_{F}\right)$ is Stein fillable, then by Theorem 3.7, $\left[M \times F, \bar{\zeta} \times \bar{\zeta}_{F}\right]=\Pi([M, \zeta])=0 \in$ $\Omega_{2 q+3}\left(B_{\zeta}^{q} \times F ; \eta_{\zeta}^{q} \oplus \zeta_{F}\right)$. The diagram then shows that that $[M, \bar{\zeta}]=0 \in \Omega_{2 q+1}\left(B_{\zeta}^{q} ; \eta_{\zeta}^{q}\right)$.

(4) If $\left(M \times F, \zeta \times \zeta_{F}\right)$ is Stein fillable then by Theorem 3.7 all $\left(\zeta \times \zeta_{F}\right)$-compatible normal $q$-smoothings of $\left(M \times F, \zeta \times \zeta_{F}\right)$ bound over $\left(B_{\zeta}^{q} \times P_{q}(F), \eta_{\zeta}^{q} \times L_{\zeta_{F}}\right)$. As a consequence,

$$
\left(\bar{\zeta} \times p_{F}\right)_{*}([M \times F])=0 \in H_{2 q+3}\left(B_{\zeta}^{q} \times P_{q}(F)\right) .
$$


Since $\left(p_{F}\right)_{*}([F]) \in H_{2}\left(P_{q}(F)\right) \cong \mathbb{Z}$ is a generator, the result now follows from the Kunneth theorem.

(5) Recall that the linking form of $M$ is a nonsingular bilinear pairing

$$
T H_{q}(M) \times T H_{q}(M) \rightarrow \mathbb{Q} / \mathbb{Z}
$$

We will show that the assumption $\bar{\zeta}_{*}([M])=0$ ensures that the linking form of $M$ vanishes, and this can only happen if $T H_{q}(M)$ vanishes.

Let $p: H_{q}(M) \rightarrow T H_{q}(M)$ be a splitting and let $p: K\left(H_{q}(M), q\right) \rightarrow K\left(T H_{q}(M), q\right)$ also denote the induced map of Eilenberg-MacLane spaces. The map $q_{M}: M \rightarrow K\left(H_{q}(M), q\right)$ inducing the identity on $H_{q}$ is such that the composition

$$
M \stackrel{q_{M}}{\longrightarrow} K\left(H_{q}(M), q\right) \stackrel{p}{\longrightarrow} K\left(T H_{q}(M), q\right)
$$

satisfies $\left(p \circ q_{M}\right)_{*}([M]) \neq 0 \in H_{2 q+1}\left(K\left(T H_{q}(M)\right), q\right)$ if $T H_{q}(M) \neq 0$ : This follows from the cohomological definition of the linking form and its nonsingularity. But since the map $\bar{\zeta}: M \rightarrow B_{\zeta}^{q}$ is a $(q+1)$-equivalence, it follows that $q_{M}$ can be factored through $\bar{\zeta}$. Hence if $\bar{\zeta}_{*}([M])=0$ then $\left(p \circ q_{M}\right)_{*}([M])=0$, the linking form of $M$ vanishes, and $T H_{q}(M)=0$.

Example 7.2. The converse of (1) in Theorem 7.1 (that $[M, \bar{\zeta}]=0 \in \Omega_{2 q+1}\left(B_{\zeta}^{q} ; \eta_{\zeta}^{q}\right)$ implies that $(M, \varphi)$ is subcritically Stein fillable) does not hold. Notice first that the adaptation of the proof breaks down, since the surgery method of Kr2 works only up to the middle dimension. Indeed, if $\Sigma \in b P_{2 q+2}$ is exotic, then $\Sigma$ admits an almost contact structure with stabilisation $\zeta$ such that $[\Sigma, \zeta]=0 \in \Omega_{2 q+1}\left(B_{\zeta}^{q} ; \eta_{\zeta}^{q}\right)$, but $\Sigma$ does not admit a subcritical Stein filling: a subcritical Stein filling of a homotopy sphere must be contractible, implying that the filling is diffeomorphic to the disk and that the homotopy sphere is standard.

Example 7.3. A simple example of a Stein fillable manifold $M$ with the property that $M \times S^{2}$ is not Stein fillable is provided by $M=S^{1} \times S^{2} \times S^{2}$ : by the fact that $M=\partial\left(S^{1} \times S^{2} \times D^{3}\right)$ we see that it is Stein fillable, while Proposition 6.2 implies that $S^{1} \times S^{2} \times S^{2} \times S^{2}$ is not Stein fillable.

Theorem 7.1 shows that the existence of a subcritical filling of $(M, \varphi)$ places strong constraints on the topology of $M$. We next pursue this point further for simply connected manifolds in dimensions 5 and 7 . Let $S^{3} \widetilde{\times} S^{2}$ and $S^{5} \widetilde{\times} S^{2}$ be the total spaces of the nontrivial linear $n$-sphere bundle over the 2 -sphere, $n=3,5$.

Proposition 7.4. Suppose that $(M, \varphi)$ is a simply connected almost contact manifold of dimension 5 or 7 and that $(M, \varphi)$ admits a subcritical Stein filling.

(1) If $M$ has dimension 5, then there is a nonnegative integer $r$ such that $M$ is diffeomorphic to one of the connected sums

$$
\sharp_{r}\left(S^{3} \times S^{2}\right) \text { or }\left(S^{3} \widetilde{\times} S^{2}\right) \sharp_{r}\left(S^{3} \times S^{2}\right),
$$

depending on whether $M$ is spin or not.

(2) If $M$ has dimension 7 and $\pi_{2}(M)$ is torsion free, then there are non-negative integers $r, s$ such that $M$ is diffeomorphic to one of the connected sums

$$
\sharp_{r}\left(S^{5} \times S^{2}\right) \sharp_{s}\left(S^{4} \times S^{3}\right) \quad \text { or }\left(S^{5} \widetilde{\times} S^{2}\right) \sharp_{r}\left(S^{5} \times S^{2}\right) \sharp_{s}\left(S^{4} \times S^{3}\right),
$$

depending on whether $M$ is spin or not. 
Proof. If $(W, J)$ is a subcritical filling of $(M, \varphi)$, then $W$ is obtained from $M$ by attaching $(q+2)$-handles and higher. It follows that the map $M \rightarrow W$ is a $(q+1)$-equivalence. Now by Theorem 7.1 (1), (3), (4) and (5), $T H_{q}(M)=0$ and so $T H_{q}(W)=0$. Since $W$ is also a simply connected manifold consisting only of handles of dimension $q$ or less, we conclude the following: if $q=2$, it follows the $W$ is homotopy equivalent to a wedge of 2 -spheres and if $q=3$, then $W$ is homotopy equivalent to wedge of 2 -spheres and 3 -spheres. Note that for the case $q=3$ we use the assumption that $\pi_{2}(M) \cong H_{2}(M)$ is torsion free. It follows, using the terminology of Wa2 that the manifold $W$ is then a stable thickening of a wedge of spheres. By [Wa2, Propsition 5.1] stable thickenings are classified up to diffeomorphism by their homotopy type and the map classifying their stable tangent bundle. Now for the $W$ we consider, $[W, B S O] \cong H^{2}\left(W ; \mathbb{Z}_{2}\right)$, the bijection being given by the second Stiefel-Whitney class. If $\downarrow$ denotes the boundary connected sum of manifolds with boundary and $D^{4} \widetilde{\times} S^{2}$ and $D^{6} \widetilde{\times} S^{2}$ denote the non-trivial linear disc bundles over $S^{2}$, we deduce that $W$ is diffeomorphic to one of the following manifolds:

$$
\begin{array}{llll}
\text { Dimension 5: } & \natural_{r}\left(D^{4} \times S^{2}\right) & \text { or } & \left(D^{4} \widetilde{\times} S^{2}\right) \natural_{r}\left(D^{4} \times S^{2}\right), \\
\text { Dimension 7: } & \natural_{r}\left(D^{6} \times S^{2}\right) \sharp_{s}\left(D^{5} \times S^{3}\right) & \text { or } & \left(D^{6} \widetilde{\times} S^{2}\right) \sharp_{r}\left(D^{6} \times S^{2}\right) \sharp_{s}\left(D^{5} \times S^{3}\right),
\end{array}
$$

and the proposition follows.

We conclude this section by viewing Example 7.2 in a more general framework. Let $L_{2 q+2}^{s, \tau}(\pi)$ denote the group of units in the surgery obstruction monoid $l_{2 q+2}(\pi)$, which was defined in [Kr2, §6]. (The notation is from [Kr1, §4] and differs from [Kr2]. In addition, $L_{2 q+2}^{s, \tau}(\pi)$ may be identified with the obstruction group $L_{2 q+2}^{C}(\pi)$ of [Wa4, 17D].) The group $L_{2 q+2}^{s, \tau}(\pi)$ acts on the set of $\left(B_{\zeta}^{q}, \eta_{\zeta}^{q}\right)$-diffeomorphism classes of complex normal $q$-smoothings $\bar{\zeta}: M \rightarrow B_{\zeta}^{q}$ without changing the $\left(B_{\zeta}^{q}, \eta_{\zeta}^{q}\right)$-bordism class. That is, writing $\left(M_{+\rho}, \bar{\zeta}_{+\rho}\right)$ for the action of $\rho \in L_{2 q+2}^{s, \tau}(\pi)$ on $(M, \bar{\zeta})$, we have

$$
[M, \bar{\zeta}]=\left[M_{+\rho}, \bar{\zeta}_{+\rho}\right] \in \Omega_{2 q+1}\left(B_{\zeta}^{q} ; \eta_{\zeta}^{q}\right)
$$

For example, if $M$ is simply connected, then $L_{2 q+2}^{s, \tau}(e) \cong \mathbb{Z}$ or $\mathbb{Z}_{2}$ as $q$ is odd or even, and the action of $L_{2 q+2}^{s, \tau}(e)$ is via connected sum with $\left(\Sigma, \bar{\zeta}_{\Sigma}\right)$, where $\Sigma$ is a generator of $b P_{2 q+2}$ and $\bar{\zeta}_{\Sigma}$ is a certain a $\left(B_{\zeta}^{q}, \eta_{\zeta}^{q}\right)$-structure on $\Sigma$.

Question 7.5. Suppose that $\bar{\zeta}: M \rightarrow B_{\zeta}^{q}$ is a normal q-smoothing such that $[M, \bar{\zeta}]=0 \in$ $\Omega_{2 q+1}\left(B_{\zeta}^{q} ; \eta_{\zeta}^{q}\right)$. Under what conditions on $M$ can we deduce that there is an element $\rho \in$ $L_{2 q+2}^{s, \tau}(\pi)$ such that $\left(M_{+\rho}, \bar{\zeta}_{+\rho}\right)$ admits a subcritical Stein filling? For example, if $M$ is simply connected, is there a homtopy sphere $\Sigma \in b P_{2 q+2}$ such that $\left(M \sharp \Sigma, \bar{\zeta} \sharp \bar{\zeta}_{\Sigma}\right)$ admits a subcritical Stein filling?

\section{REFERENCES}

[Ba] H. J. Baues, Obstruction theory on homotopy classification of maps, LNM 628, Springer-Verlag, BerlinNew York, 1977.

[BEM] M. Borman, Y. Eliashberg and E. Murphy, Existence and classification of overtwisted contact structures in all dimensions, arXiv:1404.6157

[Bou] F. Bourgeois, Odd dimensional tori are contact manifolds, Int. Math. Res. Not. 2002, no. 30, 15711574 .

[Bow] J. Bowden, Exactly fillable contact structures without Stein fillings, Algebr. Geom. Topol. 12 (2012), $1803-1810$. 
[BCS1] J. Bowden, D. Crowley and A. Stipsicz, Contact structures on $M \times S^{2}$, Math. Ann. 358 (2014), no. $1-2,351-359$.

[BCS2] J. Bowden, D. Crowley and A. Stipsicz, The topology of Stein fillable manifolds in high dimensions II, in preparation, 2013.

[Br] E. Brieskorn, Beispiele zur Differentialtopologie von Singularitäten, Invent. Math. 2 (1966), 1-14.

$[\mathrm{CE}]$ K. Cieliebak and Y. Eliashberg, From Stein to Weinstein and Back: Symplectic geometry of affine complex manifolds, American Mathematical Society Colloquium Publications, Haj 59 American Mathematical Society, Providence, RI, 2012.

[CPP] R. Casals, D. Pancholi and F. Presas, Almost contact 5-folds are contact, arXiv:1203.2166

[DG1] F. Ding and H. Geiges, E8-plumbings and exotic contact structures on spheres, Int. Math. Res. Not. (2004), no. 71, 3825-3837.

[DG2] F. Ding and H. Geiges, Contact structures on principal circle bundles, Bull. Lond. Math. Soc. 44 (2012), 1189-1202.

[E11] Y. Eliashberg, Topological characterization of Stein manifolds of dimension $>2$, Internat. J. Math. 1 (1990), 29-46.

[El2] Y. Eliashberg, AIM Workshop - Contact topology in higher dimensions: Questions and open problems. Available at http://www .math.univ-toulouse.fr/ niederkr/ANR/notes_contactworkshop2012.pdf

[EKP] Y. Eliashberg, S. Kim and L. Polterovich, Geometry of contact transformations and domains: orderability versus squeezing, Geom. Topol. 10 (2006), 1635-1747.

[Et] J. Etnyre, Contact structures on 5-manifolds, arXiv:1210.5208

[Ge1] H. Geiges, Contact structures on 1-connected 5-manifolds, Mathematika 38 (1991), 303-311 (1992).

[Ge2] H. Geiges, Applications of contact surgery, Topology 36 (1997), 1193-1220.

[Ge3] H. Geiges, Contact topology in dimension greater than three, European Congress of Mathematics, Vol. II (Barcelona, 2000), 535-545, Progr. Math. 202, Birkhäuser, Basel, 2001.

[Ge4] H. Geiges, An introduction to contact topology, Cambridge Studies in Advanced Mathematics 109, Cambridge University Press, Cambridge, 2008.

[GS] H. Geiges and A. Stipsicz, Contact structures on product five-manifolds and fibre sums along circles, Math. Ann. 348 (2010), 195-210.

[Gr] J. W. Gray, Some global properties of contact structures, Ann. of Math. 69 (1959), 421-450.

[HW] B. Hajduk and R. Walczak, Constructions of contact forms on products and piecewise fibred manifolds, arXiv:1204.1692,

[Ha] A. Hatcher, Algebraic Topology, Cambridge University Press, Cambridge, 2002.

[Hi] N. Hitchin, Harmonic spinors, Advances in Math. 14 (1974), 1-55.

[Hu] D. Husemoller, Fibre bundles, Third edition, GTM 20, Springer-Verlag, New York 1994.

$[\mathrm{KM}]$ M. A. Kervaire and J. W. Milnor, Groups of homotopy spheres I, Ann. of Math. 77 (1963) 504-537.

[Kr1] M. Kreck, An extension of the results of Browder, Novikov and Wall about surgery on compact manifolds, preprint Mainz (1985). Available at http://www.map.mpim-bonn.mpg.de/Template:Kreck1985

[Kr2] M. Kreck, Surgery and Duality, Ann. of Math. 149 (1999), 707-754.

[KS] M. Kreck and S. Stolz, Some nondiffeomorphic homeomorphic homogeneous 7-manifolds with positive sectional curvature, J. Diff. Geom. 33 (1991), 465-486.

[La1] R. Lashof, Poincaré duality and cobordism, Trans. Amer. Math. Soc. 109 (1963), 257-277.

[La2] R. Lashof Ed., Problems in differential and algebraic topology, Seattle conference, 1963, Ann. Math. (2) 81, 565-591 (1965).

[Le] J. P. Levine, Lectures on groups of homotopy spheres, Algebraic and geometric topology (New Brunswick, N.J., 1983), 62-95, Lecture Notes in Math. 1126, Springer, Berlin, (1985).

[Li] P. Lisca, Symplectic fillings and positive scalar curvature, Geom. Topol. 2 (1998), 103-116.

[Lü] W. Lück, A basic introduction to surgery theory, ICTP Lecture Notes Series 9, Band 1, "School on High-dimensional manifold theory" Trieste 2001, ICTP, Trieste 2002. Available at http://131.220.77.52/lueck/publications.php

[MNW] P. Massot, K. Niederkrüger and C. Wendl, Weak and strong fillability of higher dimensional contact manifolds, Invent. Math. 192 (2013), no. 2, 287-373.

[M] J. Milnor, A procedure for killing homotopy groups of differentiable manifolds, Proc. Sympos. Pure Math., American Mathematical Society, Providence, R.I. Vol. III (1961) 39-55. 
[MNPS] E. Murphy, K. Niederkrüger, O. Plamenevskaya and A. Stipsicz, Loose Legendrians and the plastikstufe, Geom. Topol., to appear, arXiv:1211.3895.

[PP] P. Popescu-Pampu, On the cohomology rings of holomorphically fillable manifolds, Singularities II, 169-188, Contemp. Math., 475, Amer. Math. Soc., Providence, RI, 2008.

[R] D. Ravenel, Complex cobordism and stable homotopy groups of spheres, Pure and Applied Mathematics 121, Academic Press, Inc., Orlando, 1986.

[Sa] H. Sato Remarks concerning contact manifolds, Tohoku Math. J. 29 (1977), 577-584.

[Sc] R. Schultz, Composition constructions on diffeomorphisms of $S^{p} \times S^{q}$, Pacific J. Math. 42 (1972), 739-754.

[Sm] S. Smale Generalized Poincaré conjecture in dimensions greater than 4, Ann. of Math. 74 (1961), 391-406.

[Sto] S. Stolz, Hochzusammenhängende Mannigfaltigkeiten und ihre Ränder, Springer LNM 1116, 1985.

[Stn] R. E. Stong, Notes on Cobordism Theory, Mathematical Notes, Princeton University Press, University of Tokyo Press, 1968.

[Te] P. Teichner, Topological Four-manifolds with finite fundamental group, Ph.D. thesis Mainz Verlag Shaker Aachen (1992). Available at: http://math.berkeley.edu/ teichner/papers.html

[To] H. Toda, Composition methods in homotopy groups of spheres, Princeton University Press, 1962.

[Wa1] C. T. C. Wall, Classification of $(n-1)$-connected 2n-manifolds., Ann. of Math. 75 (1962), 163-189.

[Wa2] C. T. C. Wall, Classification problems in differential topology - VI, Topology 6 (1967), 273-296.

[Wa3] C. T. C. Wall, Geometrical connectivity. I, J. London Math. Soc. (2) 3 (1971), 597-604.

[Wa4] C. T. C. Wall, Surgery on compact manifolds, Second edition. Edited and with a foreword by A. A. Ranicki. Mathematical Surveys and Monographs, 69. American Mathematical Society, Providence, RI, 1999.

[We] A. Weinstein, Contact surgery and symplectic handlebodies, Hokkaido Mathematical Journal 20 (1991), $241-251$.

Math. Institut, Universität Augsburg, Universitätstr 14, 86159 Augsburg, Germany

E-mail address: jonathan.bowden@math.uni-augsburg.de

Max Planck Institut für Mathematik, Vivatsgasse 7, D-53111 Bonn, Germany

E-mail address: diarmuidc23@gmail.com

Rényi Institute of Mathematics, Reáltanoda u. 13-15., Budapest, Hungary H-1053

E-mail address: stipsicz@renyi.hu 